\title{
Comparison of coaxial higher order mode couplers for the CERN Superconducting Proton Linac study
}

\author{
K. Papke, ${ }^{1,2,}$ F. Gerigk, ${ }^{1}$ and U. van Rienen ${ }^{2}$ \\ ${ }^{1}$ European Organization for Nuclear Research (CERN), Meyrin 1217, Switzerland \\ ${ }^{2}$ Institute of General Electrical Engineering (IAE), Faculty of Computer Science \\ and Electrical Engineering (IEF), University of Rostock, Rostock 18059, Germany
}

(Received 13 March 2017; published 12 June 2017)

\begin{abstract}
Higher order modes (HOMs) may affect beam stability and refrigeration requirements of superconducting proton linacs such as the Superconducting Proton Linac, which is studied at CERN. Under certain conditions beam-induced HOMs can accumulate sufficient energy to destabilize the beam or quench the superconducting cavities. In order to limit these effects, CERN considers the use of coaxial HOM couplers on the cutoff tubes of the 5-cell superconducting cavities. These couplers consist of resonant antennas shaped as loops or probes, which are designed to couple to potentially dangerous modes while sufficiently rejecting the fundamental mode. In this paper, the design process is presented and a comparison is made between various designs for the high-beta SPL cavities, which operate at 704.4 MHz. The rf and thermal behavior as well as mechanical aspects are discussed. In order to verify the designs, a rapid prototype for the favored coupler was fabricated and characterized on a low-power test-stand.
\end{abstract}

DOI: 10.1103/PhysRevAccelBeams.20.060401

\section{INTRODUCTION}

A bunch of charged particles propagating through a cavity will not only absorb energy from the rf field in order to be accelerated, but it also creates so-called wakefields. Depending on the spectrum of the beam, the shape of the cavity, the repetition rate of the beam, and the chopping pattern, these fields can lead to a resonant build-up and potentially influence the performance of the cavity or impact the beam dynamics. Often it is necessary to install dampers, waveguides, or coaxial couplers to reduce higher order mode (HOM) effects. The latter option has been chosen for the Superconducting Proton Linac (SPL) at CERN, Geneva [1].

The superconducting part of the SPL is composed of two types of cavities operating at $704.4 \mathrm{MHz}$ in pulsed mode with geometrical beta values' of 0.65 and 1 . Some main parameters are summarized in Tables I and II.

In spite of the experience of the Spallation Neutron Source (SNS) at Oak Ridge National Laboratory (ORNL), $\mathrm{USA}^{1}{ }^{1}$ the use of HOM coupler (Fig. 1) on the cutoff tubes of the five-cell cavities is considered necessary to allow any conceivable beam pattern. The design goal of the HOM

*kai.papke@cern.ch; kai.papke@uni-rostock.de

Published by the American Physical Society under the terms of the Creative Commons Attribution 3.0 License. Further distribution of this work must maintain attribution to the author( $s)$ and the published article's title, journal citation, and DOI.

${ }^{1}$ Excessive power coupling through several HOM ports, vacuum leaks at feed-throughs of the coupler and problems with multipacting [2]. filter is to block the transmission of the fundamental mode, while transmitting potentially dangerous HOMs. These modes are characterized by significant $(R / Q)$ values, which represent the interaction between the beam and the rf-field inside the cavity.

Preceding studies $[4,5]$ of the influence of HOMs on the beam stability in the SPL identified the modes shown in Fig. 2 for the high-beta cavities, which are the focus of this paper. Information about the influence of HOMs with respect to the medium-beta cavities can be found in $[4,6]$.

One of the most important design parameters of a HOM coupler is the external quality factor $Q_{\text {ext }}$, which describes the coupling to the modes or their damping by the coupler, respectively. The external quality factor $Q_{\text {ext }}$ is related to

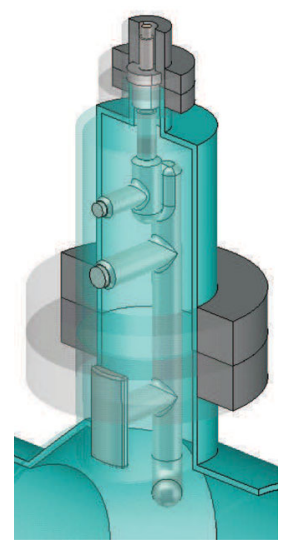

(a)

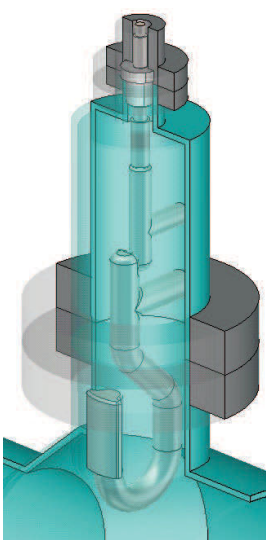

(b)

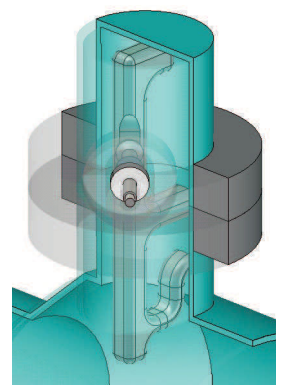

(c)
FIG. 1. Examples of HOM coupler design approaches: (a) probe coupler, (b) hook coupler, (c) modified TESLA design [3]. 
TABLE I. Nominal beam parameters for the high-power SPL.

\begin{tabular}{lccc}
\hline \hline Parameter & Units & Low-current & High-current \\
\hline Energy & {$[\mathrm{GeV}]$} & 5 & 5 \\
Beam power & {$[\mathrm{MW}]$} & 4 & 4 \\
Repetition rate & {$[\mathrm{Hz}]$} & 50 & 50 \\
Average pulse current & {$[\mathrm{mA}]$} & 20 & 40 \\
Peak pulse current & {$[\mathrm{mA}]$} & 32 & 64 \\
Source current & {$[\mathrm{mA}]$} & 40 & 80 \\
Chopping ratio & {$[\%]$} & 62 & 62 \\
Beam pulse length & {$[\mathrm{ms}]$} & 0.8 & 0.4 \\
Protons per pulse & {$\left[10^{14}\right]$} & 1.0 & 1.0 \\
\hline \hline
\end{tabular}

TABLE II. Parameters of the high-beta five-cell SPL cavity.

\begin{tabular}{ll} 
Type of accelerating structure & beta $=1,5$-cell standing wave \\
Accelerating mode & $\mathrm{TM}_{010, \pi}$ mode \\
Fundamental frequency $f_{0}$ & $704.4 \mathrm{MHz}$ \\
Nominal gradient $E_{\text {acc }}$ & $25 \mathrm{MV} / \mathrm{m}$ \\
Required field flatness $\Delta V / V$ & $< \pm 2.5 \%$ \\
Quality factor $Q_{0}$ at & $10^{10}$ \\
$\quad$ nominal gradient & \\
Active length $L$ & $1.065 \mathrm{~m}$ \\
$(R / Q)$ & $566 \Omega$ \\
$E_{\text {peak }} / E_{\text {acc }}$ & 2.0 \\
$B_{\text {peak }} / E_{\text {acc }}$ & $4.2 \mathrm{mT} / \mathrm{MV} / \mathrm{m}$ \\
Geometry factor & $270 \Omega$ \\
Number of HOM couplers & 2 \\
\hline \hline
\end{tabular}

the intrinsic quality factor of the cavity $Q_{0}$ by the coupling factor $\beta$ according to:

$$
Q_{\mathrm{ext}}=\frac{Q_{0}}{\beta}, \quad \text { with } \quad \beta=\frac{1 \pm\left|S_{11}\right|}{1 \mp\left|S_{11}\right|}=\mathrm{SWR}^{ \pm 1},
$$

with SWR as the voltage standing wave ratio. The upper sign applies in case of over-coupling whereas the lower one stands for under-coupling [7]. The recommended damping for monopole HOMs is $Q_{\text {ext }} \sim 10^{5}$ [8]. This parameter plays an important role for the adjustment (penetration depth of the coupling antenna into the cutoff tube) and will be discussed in Secs. III and VI.

This paper is organized as follows: Sec. II summarizes rf measurements carried out on copper and niobium cavities. These measurements refer to HOM spectra and the fundamental passband and confirm the results of previous simulation studies $[4,6]$. In Sec. III, the design and optimization process of the HOM coupler with respect to its rf filter characteristic is described. Seven different designs, each of them optimized for the high-beta SPL cavities, are presented. Figure 1 shows the three main design types. In Sec. IV, the heat loss as well as the extracted power is estimated. Recommendations for cooling requirements are made. A 3D-printed prototype is then

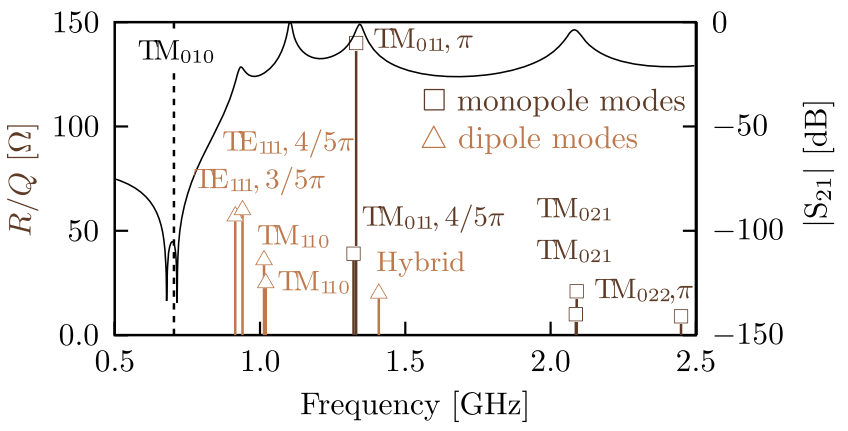

FIG. 2. HOMs with high $(R / Q)$ for the high beta SPL cavity [4,6] and a typical $S_{21}$ transmission curve (black) of an optimized HOM coupler.

presented in Sec. VI along with results of low-power measurements and comparisons with simulations. Finally, Sec. VII draws conclusions.

\section{RF MEASUREMENTS OF THE CAVITIES}

The HOM spectrum, essential for the design of a suitable HOM filter, has already been simulated and presented $[4,6]$. This section presents the first low-power measurements for the high-beta SPL cavities carried out on two copper cavities and four niobium cavities. The measurements comprise the mode frequency, the quality factor, and the field distribution which has been used to identify modes. The following types of measurements have been performed: (i) Low-power reflection and transmission type measurements $\left(S_{11}\right.$ and $\left.S_{21}\right)$ at room temperature for four niobium cavities to identify mode frequencies as well as the loaded and intrinsic quality factors $\left(Q_{L}, Q_{0}\right)$ [9,10]. (ii) Low-power transmission type measurements $\left(S_{21}\right)$ at low temperatures to evaluate frequency shifts during cooldown. (iii) Bead-pull measurements for a niobium cavity to determine the field pattern along the bead path.

The absolute values of $Q_{L}$ and $Q_{0}$ at room temperature do not give any useful information about the cavity in its superconducting state. However, the spread of those values between the fabricated cavities is of interest. The same is true for the HOM frequencies especially in terms of intercavity mode cross-talk [11]. The Q and frequency spreads also result in design requirements for the HOM coupler in relation to its sensitivity and robustness (Sec. III).

\section{A. Frequencies and quality factors}

Figure 3 summarizes the results of $Q_{0}$ measurements at warm and compares with frequency domain simulations using CST MICROWAVE STUDIO ${ }^{\circledR}$ (CST MWS) [12]. All values refer to room temperature. For the fundamental pass band the measured $Q$-values reach $>97 \%$ of the simulated values indicating an excellent surface quality with minimum 


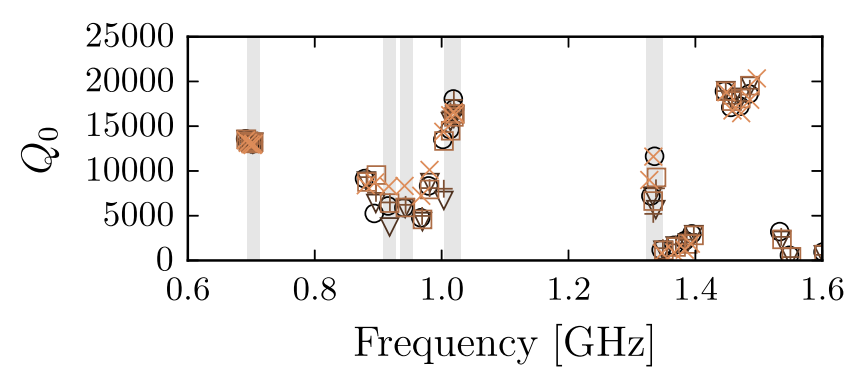

(a)

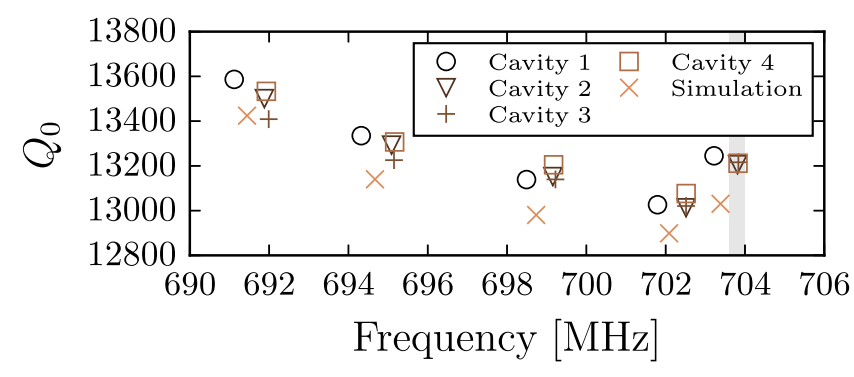

(b)

FIG. 3. Measured intrinsic quality factors $Q_{0}$ at room temperature for the four niobium SPL cavities in comparison with eigenmode simulations. The fundamental pass band is shown in detail. Modes with the $(R / Q)>10$ are highlighted.

roughness. The measured $Q_{0}$ spread of the HOMs in Fig. 3 is below $10 \%$ and is therefore very consistent between the different cavities.

Most of the modes with high $(R / Q)$ values exhibit a frequency spread of $<1 \mathrm{MHz}$ (Fig. 4). The majority of the investigated modes up to $2.1 \mathrm{GHz}$ shows a variation of less than $3 \mathrm{MHz}$. The relatively low frequency variance relaxes the requirements for the HOM coupler with respect to the needed bandwidth around the HOMs (see Sec. IIIC). Furthermore, it allows a very precise adjustment of the coupler to specific HOMs. There is a comparably high spread of the $\mathrm{TM}_{021}$ mode at around $2.09 \mathrm{GHz}$, but this is less problematic.

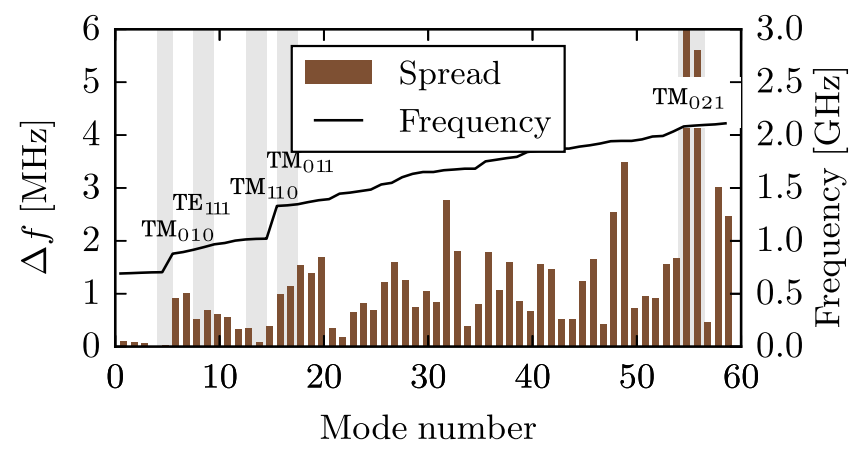

FIG. 4. Maximum frequency spread for the measured modes between 690 and $2100 \mathrm{MHz}$. Modes with highest $(R / Q)$ values are highlighted and labeled.
The frequency of modes up to $2.5 \mathrm{GHz}$ was also measured at $2 \mathrm{~K}$ in order to evaluate the frequency shift during cool-down. The results agree well with calculations based on thermal contraction according to the relation $\Delta f / f=-\Delta L / L$, with $\Delta L / L=0.143 \%$ for bulk niobium. For example, the frequency of the fundamental mode decreases by $1 \mathrm{MHz}$ [13].

\section{B. Field characterization}

The field distribution of selected modes has been analyzed with bead-pull measurements [7] to identify the modes and to assign the simulated $(R / Q)$ values as Fig. 2 to the measured modes. We use a dielectric bead, which primarily perturbs the electric field. Figure 5 shows a comparison of the measured frequency shift (proportional to the magnitude of the electric field squared) and the corresponding field pattern resulting from an eigenmode simulation.

The $\mathrm{TM}_{011} \pi$ mode at $\sim 1.33 \mathrm{GHz}$ shown in Fig. 5 is obviously concentrated in one of the end cells, which allows a good coupling by a HOM coupler on the corresponding cutoff tube.

\section{COUPLER DESIGN PROCESS AND RF CHARACTERISTICS}

The HOM coupler is designed to provide minimal transmission at the operating mode frequency while good transmission at the HOM frequencies (Fig. 2). Furthermore, the design needs to optimize the multipacting behavior, allow the heat load to be investigated, minimize the mechanical complexity, and maximize the tolerances in order to keep the cost at a reasonable level. Due to this multiphysical problem, the approach to a coupler design is quite heuristic and hence requires comparison with other approaches. We considered a total of seven designs (Figs. 6 and 7), which have been optimized in terms of rf characteristics also considering fundamental mechanical limitations (e.g. maximum height). These designs were then assessed in terms of $Q_{\text {ext }}$, heat load, monopole coupling, dipole coupling, robustness, and tunability.

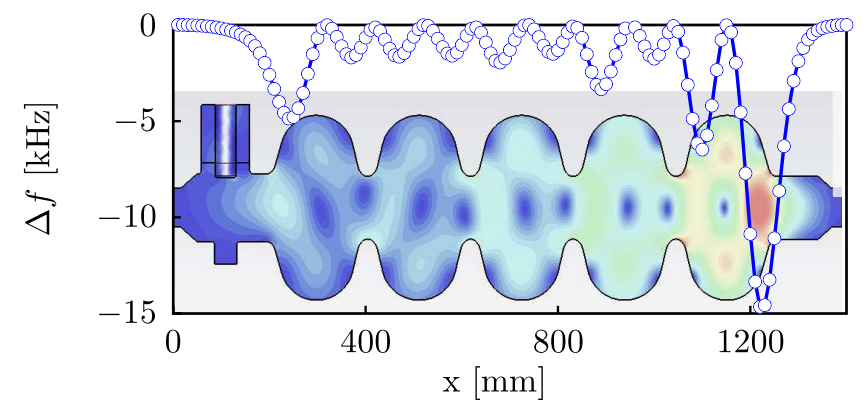

FIG. 5. Bead-pull measurement for the $\mathrm{TM}_{011} \pi$ mode $(1.333 \mathrm{GHz})$ compared with the electric field magnitude resulting from an eigenmode simulation (contour plots with dark blue representing areas of low field strength). 


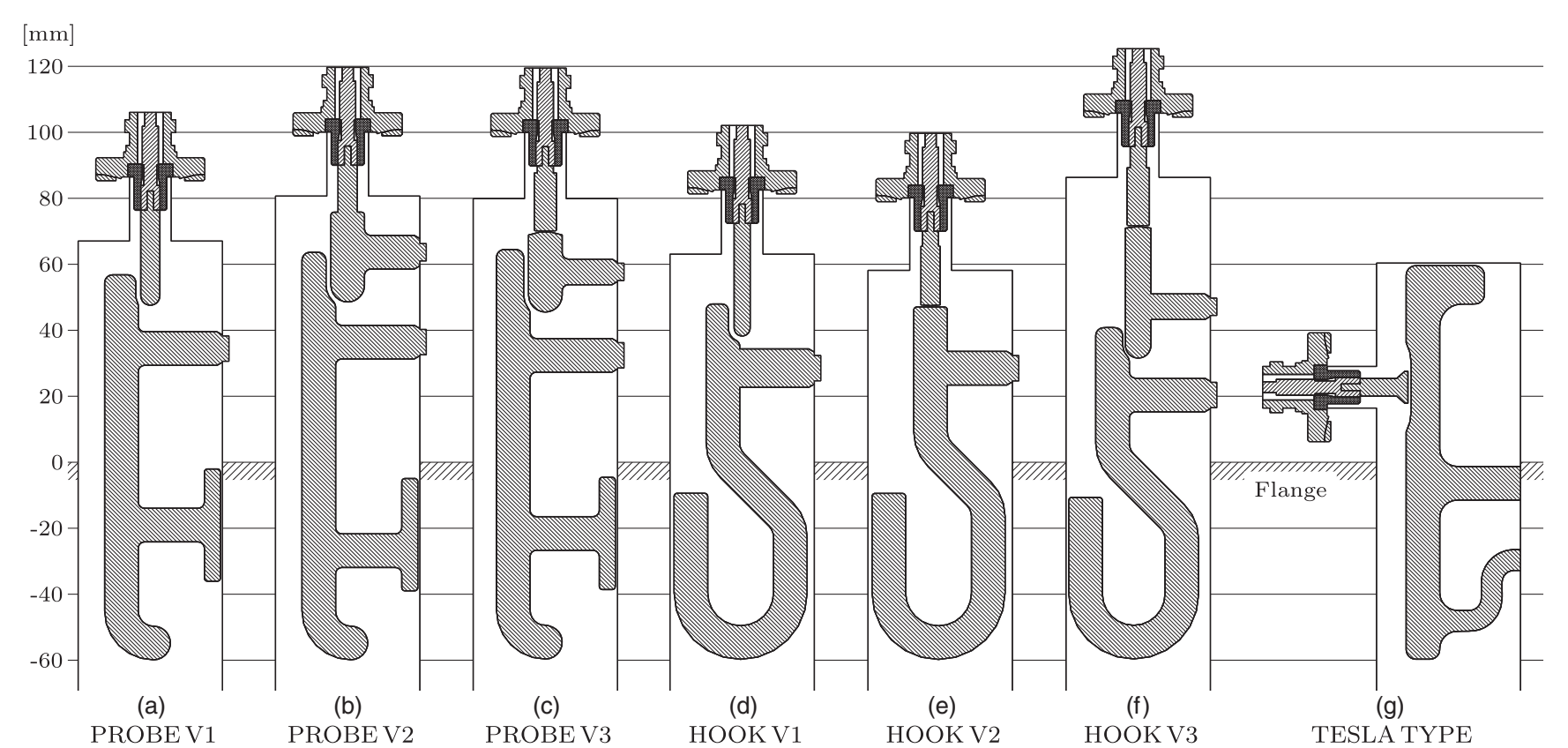

FIG. 6. HOM coupler design approaches for the high-beta SPL cavity, the dashed line indicates the flange. In the following "stages" refers to parts of the couplers separated by gaps. (a) Probe Coupler Version 1 with single notch filter, two stages, and centered output port. (b) Probe Coupler Version 2 with double notch filter, two stages, and centered output port. (c) Probe Coupler Version 3 with double notch filter, three stages, and centered output port. (d) Hook Coupler Version 1 with single notch filter, two stages, and centered output port. (e) Hook Coupler Version 2 with single notch filter, two stages, and shifted output port. (f) Hook Coupler Version 3 with double notch filter, three stages, and centered output port. (g) Modified TESLA design.

\section{A. Design process}

We start the design process with a synthesis of the rf filter characteristics. The PROBE V3 coupler (Fig. 7) serves as reference in the following. For analyzing and optimizing each coupler design, simplified equivalent circuit models have been used (see Fig. 8). The electrical circuit consists of two interlinked parts: (i) The (primary) notch filter ( $\mathrm{Ln}, \mathrm{Cn}$ ) with a resonance frequency equal to the operating frequency of the cavity in order to reject the fundamental mode. (ii) A high pass filter, for the transmission of the HOMs which can be modeled by coupled resonant circuits. The first part is not always mandatory because it can also be part of the HOM transmission network as in Fig. 8 (secondary notch). Here, in fact, the inductance L3 is caused by scattering effects of M23 and M34 which are high enough to create a parallel circuit with $\mathrm{C} 3$ resonating at around $704 \mathrm{MHz}$ and forming the (secondary) notch filter. However, depending on the filter order, the rejection of the fundamental mode might not be sufficient. In this case an additional notch filter is necessary to increase the rejection and/or to provide a larger bandwidth of the notch filter. A second order Chebyshev high pass filter as for example considered in [14], does not necessarily require an additional notch filter. In contrast, filters with an order greater than one always yield more complex designs, such as the PROBE V2, V3 and the HOOK V3 coupler in Fig. 6.

Additionally, the design needs to take into account the ceramic window at the feed-through with its relative high capacitance. This capacitance (C5 shown in Fig. 8) impacts notably the matching to the $50 \Omega$ load (impedance of the rf cable).

The capacitors and inductances of the lumped circuit model are determined with respect to the fundamental mode as well as the HOM spectrum [4]. Finally, the lumped circuit model will be converted into an equivalent transmission line (TML) circuit which defines the different lengths of the coupler parts at one frequency $[15,16]$. This frequency has to
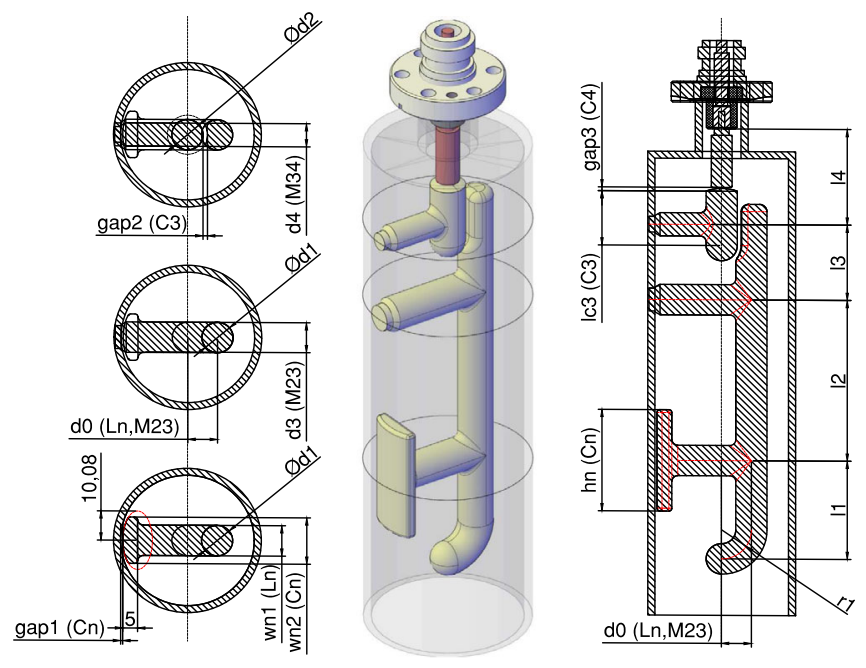

FIG. 7. Parametrized model of the PROBE V3 coupler. 
be a middle frequency of the considered HOMs so that we obtain an estimation of the part lengths rather than optimal parameters for the 3D model. This approximation is then refined using 3D simulation tools such as CST MWs [12] and ANSYS ${ }^{\circledR}$ HFSS [17]. Figure 9 compares the transmission behavior of the PROBE V3 coupler simulated by HFSS with its equivalent transmission line model (Figs. 7 and 8). The TML model describes reasonable well the relationship between the coupler geometry and the filter characteristics of the coupler, particularly in the passband of the filter (>900 MHz). The effect of parameter 12 on the notch filter (Fig. 9) however, is not covered by the simplified model as it does not foresee any coupling between the inductances Ln and L2.

In all designs the following mechanical limitations and preferences apply: (i) The tube diameter is fixed to $45 \mathrm{~mm}$ and the diameter of the HOM antenna should be as large as possible to simplify the fabrication of a hollow antenna which can be actively cooled by liquid helium. A larger diameter reduces the space inside the tube, which decreases the scope of inductances (Ln, M23, M34 in Fig. 8). A diameter of $10.5 \mathrm{~mm}$ was chosen as a compromise. (ii) The distance between flange and cutoff tube is $46 \mathrm{~mm}$ and the coupler should penetrate the cutoff tube in a range between 0 and $15 \mathrm{~mm}$. This limits, for example, the lengths 11 and 12 in the transmission line model in Fig. 8. (iii) The maximum length of the coupler is $150 \mathrm{~mm}$ measured from the flange to the rf connector.

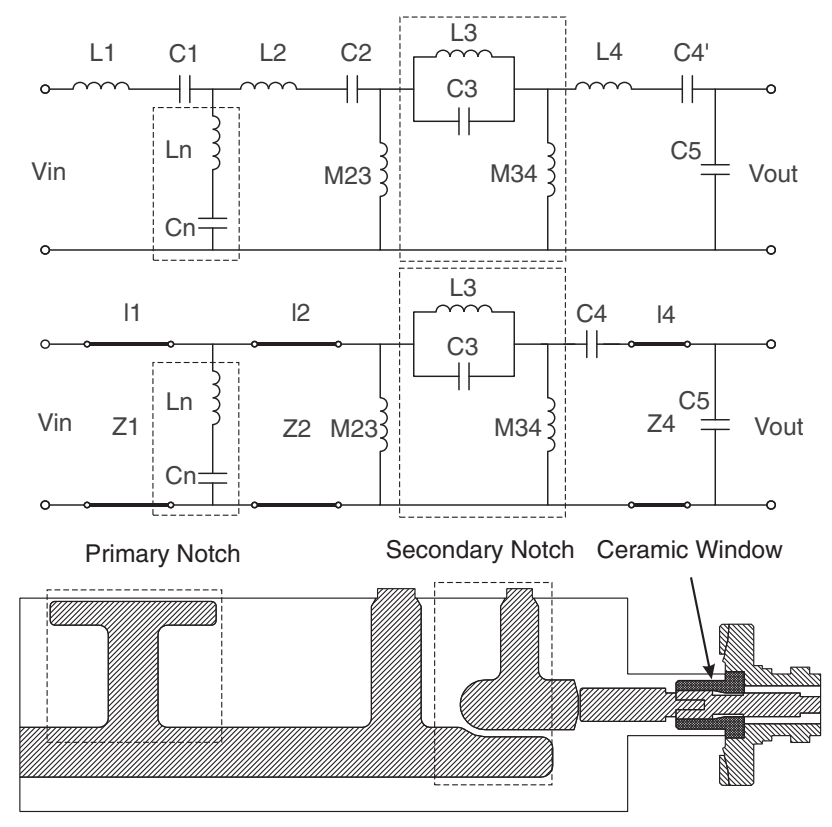

FIG. 8. Equivalent lumped circuit and transmission line model of the PROBE V 3 design in comparison to the cross section of the three dimensional model. Only the filter is considered, not the antenna penetrating the beam pipe.
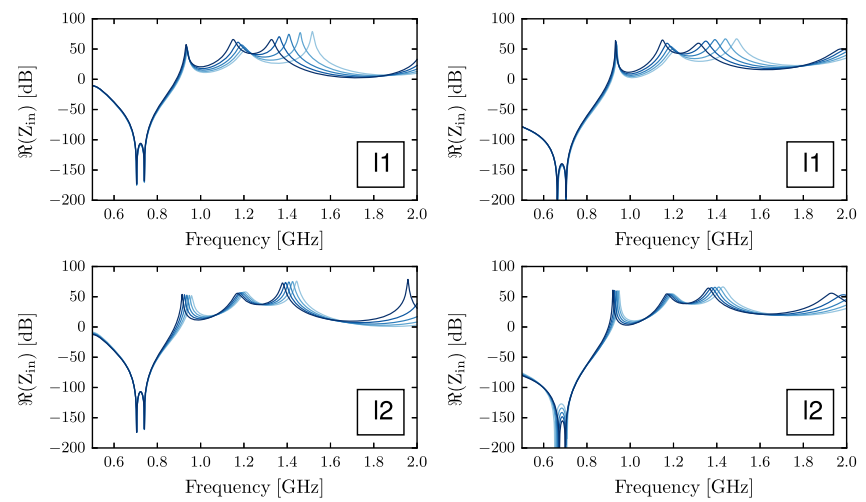

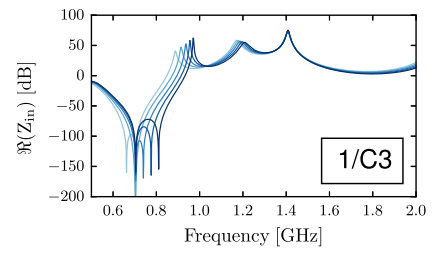

(a)

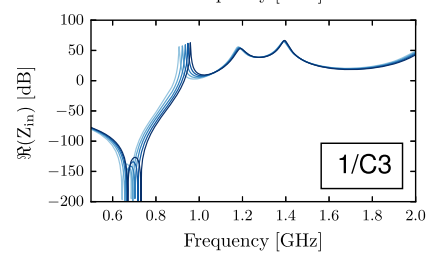

(b)
FIG. 9. Input impedance of the transmission line equivalent circuit model (a) and of the corresponding 3D model simulated by HFSS (b) assuming a $50 \Omega$ load at the output (Fig. 8). Three parameters of the model were varied in a range between $-20 \%$ (light blue) and $+20 \%$ (dark blue) from the nominal value. The parameter $(1 / \mathrm{C} 3)$ is proportional to the gap size of the capacitor (gap2 in Fig. 7).

\section{B. Optimization and transmission behavior}

In order to optimize the pure transmission characteristic, the HOM couplers were mounted on a tapered beam pipe section for the simulation (Fig. 1). Terminating the ends of the beam pipes with waveguide ports allows the excitation of monopole as well as dipole modes, whereas a TEM mode is transmitted out of the coaxial output of the coupler. For a faster optimization, the HOM coupler tube can be directly terminated by a port. The results concerning the resonances of the $S_{21}$ curve are nearly the same as for the more complex and time consuming models. In both cases basically two S-parameters are of interest: the $\mathrm{TM}_{01}$-TEM transmission describing monopole coupling and the $\mathrm{TE}_{11^{-}}$ TEM transmission describing the dipole coupling (Fig. 10).

Taking into account potentially dangerous HOMs (Fig. 2), the optimization of the initial design coming from the TML model has been subjected to the following priorities: 1. Rejection of the fundamental mode at $704.4 \mathrm{MHz}$ (adjustment of the notch filter). 2. Transmission of the $\mathrm{TM}_{011}$ monopole modes at around $1.33 \mathrm{GHz}$ with $(R / Q)$ up to $140 \Omega$. 3. Optional: Transmission of the $\mathrm{TE}_{111}$ dipole modes at around $940 \mathrm{MHz}$ with $(R / Q)$ up to $60 \Omega$. The optimization incorporates numerous tuning parameters such as those shown in Fig. 7. Some of them are not part of the TML model (e.g. hook radius $r 1$ ). It is preferable to first adjust the mutual inductances M2 3 and M34 (parameter $\mathrm{d} 3$ and $\mathrm{d} 4$ in Fig. 7) to achieve a similar passband behavior of the filter as 


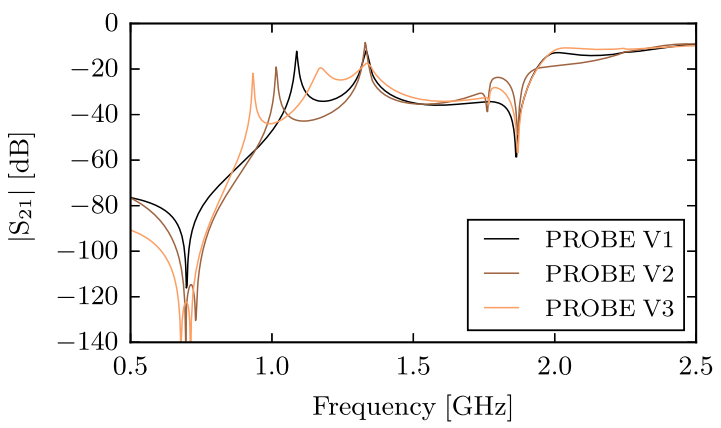

(a)

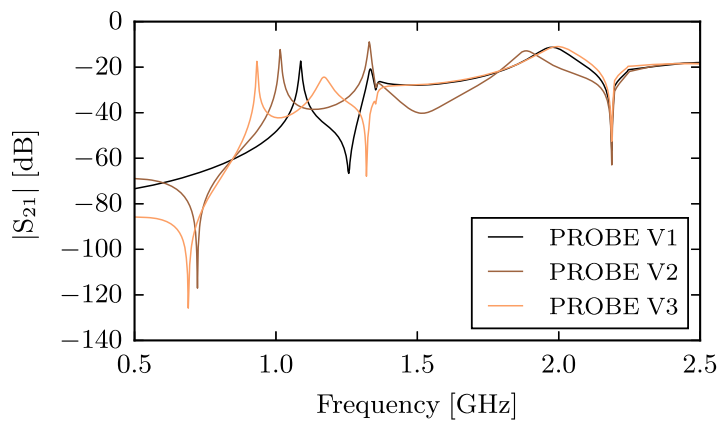

(c)

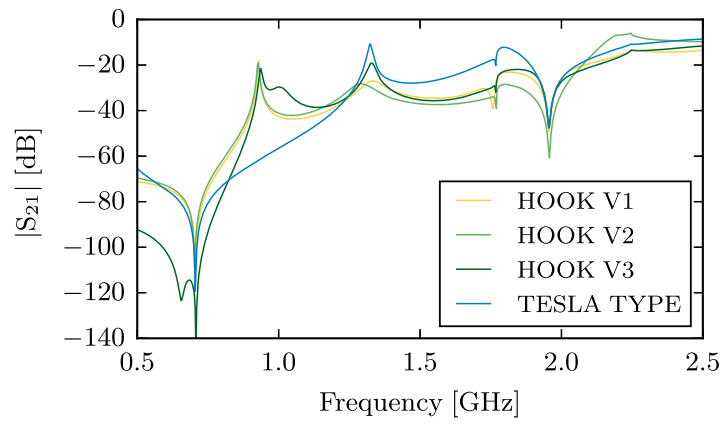

(b)

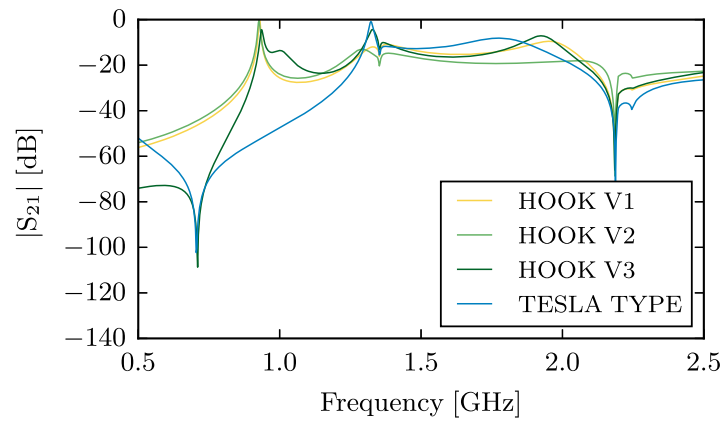

(d)

FIG. 10. (a) and (b) $\mathrm{TM}_{01}$-TEM transmission (monopole coupling) of the different design approaches optimized for the high-beta SPL cavity. (c) and (d) $\mathrm{TE}_{11}$-TEM transmission (dipole coupling for the most favorable polarization) of the different design approaches optimized for the high-beta SPL cavity.

predicted by the TML model. A horizontal displacement of the antenna by the parameter do allows higher inductances without greatly reducing the diameters $\mathrm{d} 3$ and $\mathrm{d} 4$. Afterwards, the lengths 11 and 12 can be tuned. They have the main influence on the passband resonances. The primary notch filter is mainly determined by the gap (gap1) and the plate size $(\mathrm{wn} 2 \times \mathrm{hn})$. The gap distance ranges between 0.7 and $1.0 \mathrm{~mm}$ for the considered designs. The secondary notch is mainly influenced by the length 13 as well as the gap size of C3 (gap2) which is approximately $1.5 \mathrm{~mm}$. The capacitor $\mathrm{C} 4$ and the length 14 affect the matching to the $50 \Omega$ load. By changing these two parameters, the resonances in the passband of the filter (Fig. 9) become either more pronounced or smoother and more damped. This procedure has to be repeated several times as many parameters affect each other. Also, the radius of the HOM antenna (d1) is optimized at this stage.

The results for the rf optimized couplers including the beam pipe section are shown in Fig. 10. The notches in the upper frequency range (1.7 to $2.3 \mathrm{GHz}$ ) of the transmission curves correspond to cutoff frequencies of the related traveling modes in the beam pipe section. The HOM coupler has a negligible influence on those resonances. Furthermore, no dangerous HOMs are expected for the high-beta SPL cavities in this frequency range.

The different design approaches can be classified according to (i) the number of notches to reject the fundamental mode, which corresponds to the filter order, (ii) the type of coupling (loop or probe antenna).
The first criterion is linked to the complexity of the coupler. The designs PROBE V2, V3, and HOOK V3 in Fig. 6 feature two notches, which provide a higher attenuation over a considerably larger frequency range. The bandwidths of the double and single notch filters are summarized in Table III and range from a few $\mathrm{MHz}$ up to more than $200 \mathrm{MHz}$ at $-100 \mathrm{~dB}$. Besides the better rejection, those couplers with higher filter order also show a steeper transition between stop and passband. Due to this fact, modes of the very first HOM band (1st dipole band at $\sim 920 \mathrm{MHz}$ ) can already be damped more efficiently as shown in Fig. 10(c) and (d). Moreover, the filter order also corresponds to the maximum possible number of resonances in the passband to enable the damping of specific HOMs. However, the use of more than one notch results in a more challenging fabrication of the coupler. More welds are required which increases the risk of vacuum leaks and also the cleaning becomes more difficult.

The second criterion to classify HOM couplers is related to the shape of the coupling antenna, which features either a dominating electric (probe antenna, first three designs in Fig. 6) or magnetic [loop antenna, couplers (d) to (f) in Fig. 6] coupling. The TESLA design [2,3,18] [Fig. 6(g)] mostly couples to the magnetic field and can therefore be called a loop coupler, but this depends on the shape of the probe extremity. The longer this extremity, the stronger is the electric coupling compared to the magnetic one.

The modified TESLA design has a very narrow notch filter, which is however much easier to tune than for other 
TABLE III. Sensitivity of the frequency transmission corresponding to the gap variation of the notch(es) by $\pm 0.3 \mathrm{~mm}$.

\begin{tabular}{|c|c|c|c|c|c|c|c|}
\hline $\begin{array}{l}\text { Coupler } \\
\text { design }\end{array}$ & Parameter & $\begin{array}{c}\text { 1st Notch } \\
\text { sensitivity } \\
{[\mathrm{MHz} / \mathrm{mm}]}\end{array}$ & $\begin{array}{c}\text { 2nd Notch } \\
\text { sensitivity } \\
{[\mathrm{MHz} / \mathrm{mm}]}\end{array}$ & $\begin{array}{c}\text { Minimum damping } \\
\text { sensitivity } \\
\text { in the stop } \\
\text { band }[\mathrm{dB}]^{\mathrm{a}}\end{array}$ & $\begin{array}{c}\text { Resonance sensitivity } \\
\text { at around } 940 \mathrm{MHz} \\
\text { dipole band } \\
{[\mathrm{MHz} / \mathrm{mm}]^{\mathrm{b}}}\end{array}$ & $\begin{array}{c}\text { Resonance sensitivity } \\
\text { at around } 1.3 \mathrm{GHz} \\
\text { monopole band } \\
{[\mathrm{MHz} / \mathrm{mm}]^{\mathrm{c}}}\end{array}$ & $\begin{array}{c}\text { Notch } \\
\text { bandwidth } \\
\text { at }-100 \mathrm{~dB} \\
{[\mathrm{MHz}]}\end{array}$ \\
\hline PROBE V1 & gap1 & 383.77 & $\ldots$ & $\ldots$ & $\ldots$ & 16.88 & $5.0 \ldots 25.0$ \\
\hline \multirow[t]{2}{*}{ PROBE V2 } & gap1 & 246.67 & 138.01 & $-91.52 \ldots-115.38^{\mathrm{d}}$ & $\ldots$ & 62.05 & $85.0 \ldots 180.0$ \\
\hline & gap2 & 129.29 & 7.08 & $-84.67 \ldots-111.42$ & $\ldots$ & 32.92 & $85.0 \ldots 172.5$ \\
\hline \multirow[t]{2}{*}{ PROBE V3 } & gap1 & $180.74 *$ & $177.71 *$ & $-121.93 \ldots-98.71^{\mathrm{d}}$ & 42.75 & 19.81 & $135.0 \ldots 215.0$ \\
\hline & gap2 & $70.84 *$ & $47.73 *$ & $-106.71 \ldots-121.49^{\mathrm{d}}$ & 84.94 & 1.75 & $137.5 \ldots 155.0$ \\
\hline HOOK V1 & gap1 & 155.74 & $\ldots$ & $\ldots$ & -6.71 & -10.50 & $<0.1 \ldots .5 .0$ \\
\hline HOOK V2 & gap1 & 143.83 & $\ldots$ & $\ldots$ & -5.74 & -12.55 & $2.5 \ldots 7.5$ \\
\hline \multirow[t]{2}{*}{ HOOK V3 } & gap1 & 251.93 & -5.27 & $-101.86 \ldots-122.84$ & 13.56 & -8.60 & $130.0 \ldots 220.0$ \\
\hline & gap2 & $126.75 *$ & $136.00 *$ & $-128.86 \ldots-106.62^{d}$ & 144.50 & 161.83 & $137.5 \ldots 160.0$ \\
\hline TESLA & gap1 & 692.53 & $\ldots$ & $\ldots$ & $\ldots$ & 19.73 & $5.0 \ldots 17.5$ \\
\hline
\end{tabular}

" ${ }^{\text {a } O n l y ~ g i v e n ~ f o r ~ d e s i g n s ~ w i t h ~ d o u b l e ~ n o t c h ~ f i l t e r . ~ T h e ~ p a r a m e t e r ~ c o r r e s p o n d s ~ t o ~ t h e ~ m a x i m u m ~ o f ~ t h e ~} S_{21}$ curve between the two notches around 704.4 MHz [Figs. 10(a) and (b)].

${ }^{\mathrm{b}}$ Parameter corresponds to the first maximum in the passband, tuned to modes of the $\mathrm{TE}_{111}$ band [Figs. 10(c) and (d)]. For the Probe $\mathrm{V} 1$ and V2 couplers, this resonance deviates strongly from $940 \mathrm{MHz}$ due to limited optimization (see Sec. III B).

${ }^{\mathrm{c}}$ Depending on the design the parameter corresponds to the second or third maximum in the passband of the coupler which is around $1.33 \mathrm{GHz}$ [Figs. 10(a) and (b)].

${ }_{\mathrm{d}}^{\mathrm{A}}$ linear interpolation is only valid for parameter variations $<0.1 \mathrm{~mm}$. Otherwise a second order interpolation is required.

couplers [19]. A notch filter close to the beam pipe concentrates the surface loss of the fundamental mode in the HOM coupler mainly at the coupling antenna. In case of the TESLA design, which features the notch filter on the opposite side as far as possible from the beam pipe, the field is slightly less concentrated (Sec. IVA) than for the other designs. In the end, the TESLA coupler had to be excluded for the SPL cavity due to mechanical restrictions. The design shown in Fig. 6(g) is not feasible, because both fixings are below the flange whose position is marked by the "zero" level. Shifting these fixings above the flange would spoil the whole if performance. The only solution would be to weld the fixings onto the port, but then the HOM couplers would no longer be removable. Hence, we consider the modified TESLA design only for comparison but not as an option for the SPL.

The hook designs are characterized by their dipole coupling (especially for the first dipole band at $\sim 920 \mathrm{MHz}$ ), whereas the probe designs provide a better damping of the $\mathrm{TM}_{011}$ modes at $1.33 \mathrm{GHz}$. From all the considered design approaches, the PROBE V3 and the HOOK V3 coupler [Fig. 6(c) and (d)] achieve the best rf performance also in terms of robustness as discussed in Sec. III C.

\section{Sensitivity analysis}

The mechanical tolerance of a coupler should not be smaller than $\pm 0.2 \mathrm{~mm}$ to limit the cost of fabrication. The most sensitive parts are the capacitors of the notch filter (Fig. 8). The corresponding capacitive gaps are usually less than $1 \mathrm{~mm}$, which causes the high mechanical sensitivity. In case of the single notch designs (e.g. PROBE V1 in Fig. 11), the sensitivity is very high, which makes a precise tuning of the installed coupler mandatory. In contrast, the tuning of double notch HOM couplers is much easier or even not needed if a tolerance of $\pm 0.2 \mathrm{~mm}$ is kept.

A detailed sensitivity analysis has been carried out using the simulation models mentioned in Sec. III B including the tapered beam pipe section. In doing so, the gap distances of the notch filter have been varied in a range of $\pm 0.3 \mathrm{~mm}$ with a step size of $\pm 0.03 \mathrm{~mm}$ in order to analyze the dependency (linear or nonlinear) of the notch filter frequency on the gap width. Besides the impact on the notch resonances, the effect on the damping limits in the stop and passband, the influence on resonances in the passband as well as the band width variation of the notch filter have been recorded as shown in Table III. The results clearly underline the higher robustness

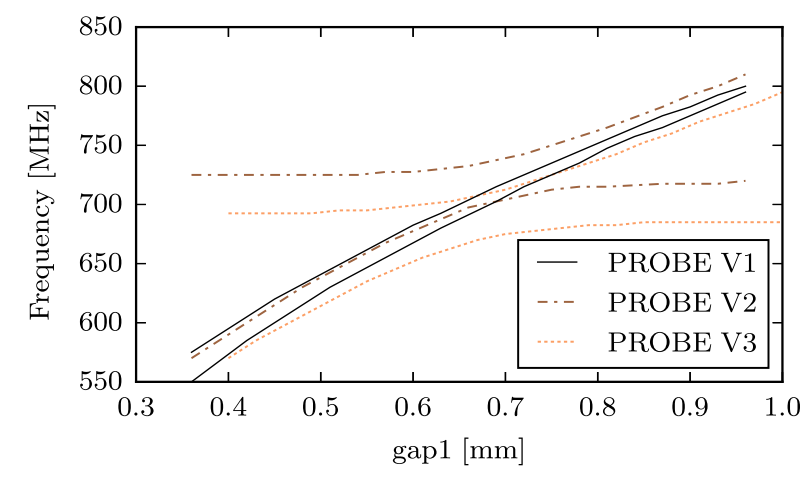

FIG. 11. Notch bandwidth as a function of the gap distance of the primary notch (compare Fig. 8). For the coupler with a single notch filter (PROBE V1), the bandwidth is measured at $-100 \mathrm{~dB}$. For the couplers with double notches (PROBE V2, V3), it is measured via the distance between both notch frequencies. The more they deviate the less damping will be achieved in between and they become separate single notch filters. 
of double notch designs. Most of the dependencies appear to be linear in the considered parameter range.

Figure 11 compares the probe couplers in terms of the notch filter frequency as a function of the gap distance.

\section{External $Q$ simulations}

Finally, the damping of the HOMs in the SPL cavity has been studied by eigenmode simulations using CST MWS. For this purpose, two HOM couplers have been considered, one on each end of the cavity with a penetration depth of $20 \mathrm{~mm}$. The cutoff tubes have diameters of 135 and $140 \mathrm{~mm}$. The results are shown in Fig. 12 for the first two dipole bands $\left(\mathrm{TM}_{011}, \mathrm{TM}_{021}\right) . Q_{\text {ext }}$ values range between $10^{3}$ to $10^{6}$.

In Sec. VI, this kind of simulation is compared with prototype measurements in order to confirm the simulated values of $Q_{\text {ext }}$. Figure 12 illustrates the different damping characteristics of the hook and probe antennas. The hook couplers provide higher damping of the dipole modes while the probe couplers provide a better damping of the monopole modes around $1.33 \mathrm{GHz}$. The simulation results give an indication of the expected order of magnitude for the external $Q$ values. Eventually, the penetration depth

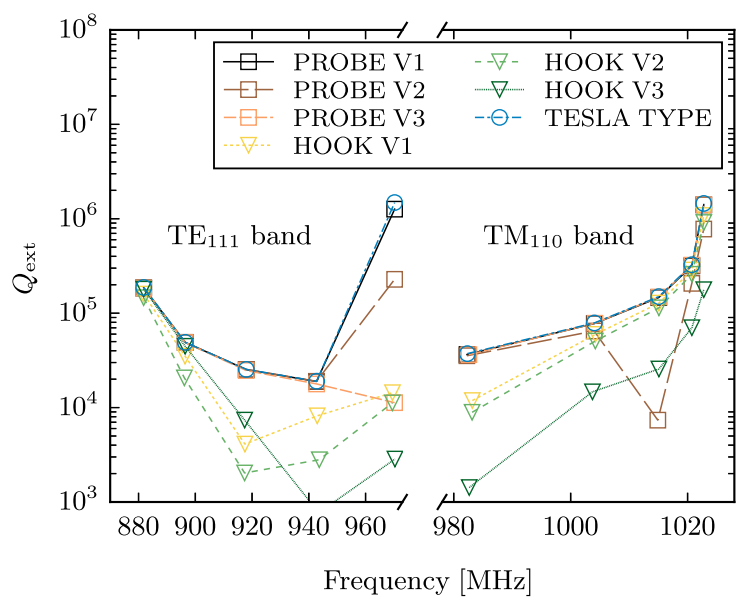

(a)

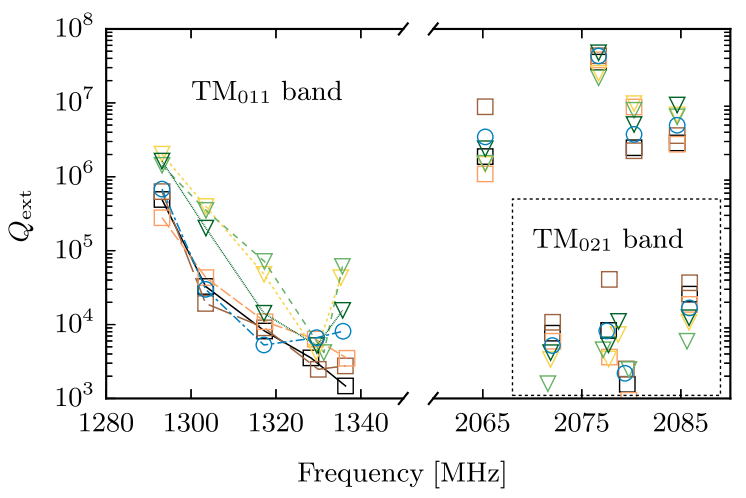

(b)

FIG. 12. Simulated $Q_{\text {ext }}$ considering a HOM coupler on both sides of the cavity. (a) Modes of the 1 st and 2 nd dipole bands. (b) Modes of the $\mathrm{TM}_{011}$ and $\mathrm{TM}_{021}$ monopole bands. of the coupler has to be adjusted in order to reduce or increase the damping. Deeper penetration of the coupler would result in stronger damping, however the coupler may incur higher rf heating, which may cause the cavity to quench.

\section{THERMAL ANALYSIS}

A number of thermal and coupled rf-thermal simulations have been carried out in order to define cooling requirements that ensure a stable superconducting state of the coupler surface. In contrast to normal conducting applications, we are more interested in peak temperatures rather than the average heat loss. Once the coupler quenches and becomes normal conducting, the dissipated power in the surface rises by several orders of magnitude, which could potentially burn and melt parts of the coupler [2,20]. We consider two different heat sources for the HOM coupler, which act on the input and output side: (i) Power dissipation caused by the electromagnetic fields in the coupler (extracted from the cavity). (ii) Heat transfer via the rf cable, which extracts the HOM power. Depending on the operating mode of the cavity and on the number of excited HOMs, the power dissipation may vary widely. Hence, it is not necessarily dominating the overall thermal load. However, this part is more difficult to estimate as it depends on beam excited modes and on the surface resistance which is strongly dependent on the surface preparation. Both aspects require measurements to make a reasonable estimate of the dissipated power. We therefore consider different case scenarios for the simulations and deduce from those results a temperature range at which the couplers operate.

Likewise, the second heat source (heat transfer) should not be underestimated. A certain cable length inside the cryo module (Fig. 13) and the use of heat intercepts may reduce the heat transfer to the coupler significantly. In the following we discuss both aspects.

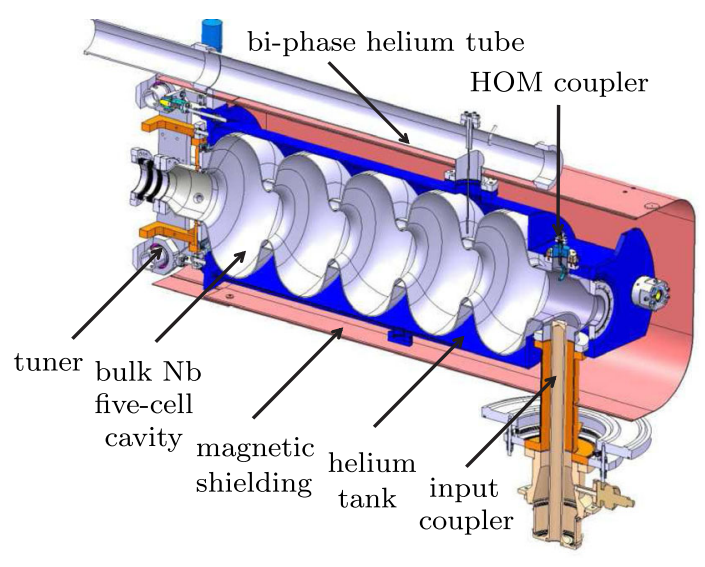

FIG. 13. Schematic sectional view of a five-cell SPL cavity inside its helium tank [1]. HOM couplers are on both sides of the cavity (left one is hidden). 


\section{A. Rf heating}

The analysis is split in two parts, the rf simulation and the subsequent steady state thermal simulation. The first provides the surface current on the cavity wall which is related to the heat flux density in the thermal simulation via the surface resistance. The uncertainty of the surface resistance as discussed in the following preclude a precise evaluation of the coupler temperature. Instead, we propose to evaluate the temperature ranges in correlation to the surface resistance in which the coupler can operate stably.

\section{1. $R f$ simulation}

We first consider the electromagnetic field of the fundamental mode and HOMs with high $(R / Q)$-values calculated for a 3D model including the 5-cell cavity, two HOM couplers and the power coupler (Fig. 13). For each mode we may define an electric current on the coupler surface according to:

$$
I_{i}^{2}=\oiint_{\partial \boldsymbol{\Omega}_{\text {coupler }}} \mathbf{H}_{i} \cdot \mathbf{H}_{i}^{*} d A,
$$

where the index zero corresponds to the fundamental mode. Figures 14 and 15 represent a selection of field patterns for different coupler designs and modes calculated by ANSYS HFSS.

The surface current and dissipated power are connected via the surface resistance composed by $R_{\mathrm{BCs}}$ according to the BCS theory [21] and $R_{\text {res }}$, the temperature-independent residual resistance [22]. A computer code originally written by Halbritter [23] is used to calculate the temperature dependent part of the surface resistance up to the critical temperature. The residual resistance for bulk niobium is calculated by (2.27) in [22] showing a quadratic

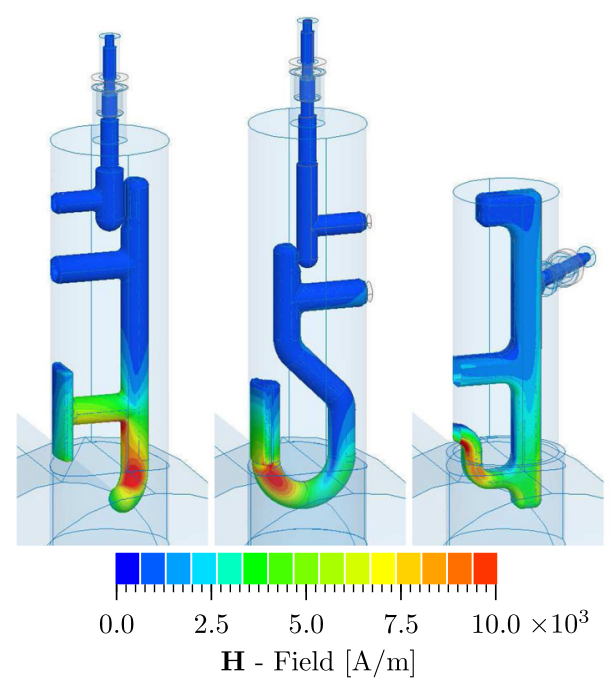

FIG. 14. Magnetic field strength $\mathbf{H}$ of the fundamental mode of different couplers. The field is scaled to a gradient of $25 \mathrm{MV} / \mathrm{m}$ in the 5-cell cavity.

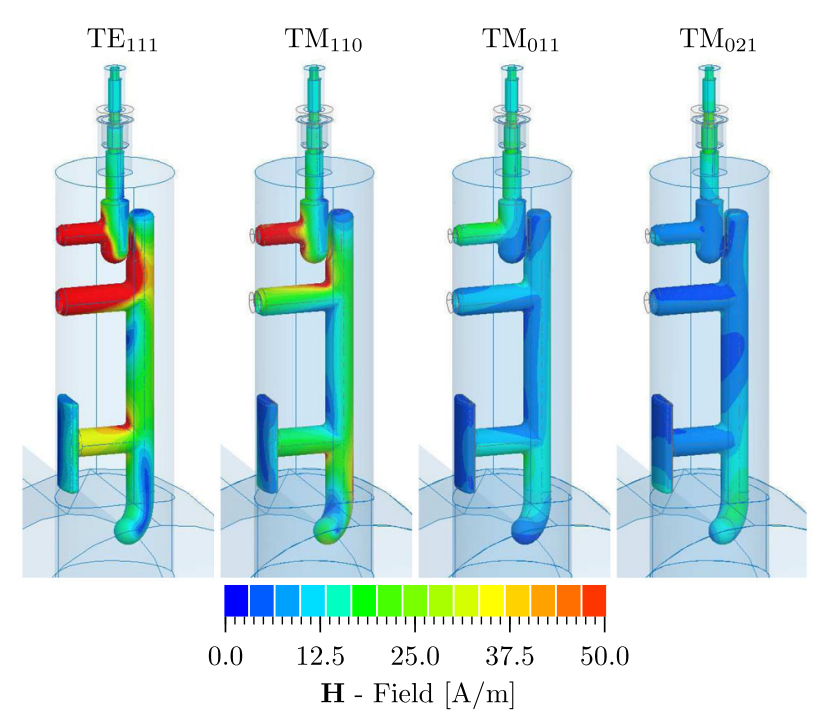

FIG. 15. Magnetic field $\mathbf{H}$ of different higher order modes in case of the PROBE V3 coupler. The fields are scaled to an extracted power of $1 \mathrm{~W}$.

dependency on the frequency. From the measurements presented in [24], $R_{\text {res }}$ can be estimated to a few nanoohms, hence, is a minor contribution to the total surface resistance that we will consider later. The calculations are limited by our incomplete knowledge of the material properties represented by the residual resistance ratio RRR [7]. The latter depends on surface treatments, local impurities and welds [20]. For the sake of simplicity, we assume homogeneously distributed surface properties.

We introduce a free variable $R_{0}$ which defines the homogenous surface resistance at $704.4 \mathrm{MHz}$. Later on, we will investigate the correlation between this parameter and the coupler temperature for different RRR in order to derive possible thermal conditions. The local surface density of the dissipated power, $\partial P_{\text {dissipated }} / \partial A$, serves as input for the heat analysis. As we consider a limited number of modes we approximate the total heat flow on the coupler surfaces as follows:

$$
\frac{\partial P_{\text {dissipated }}}{\partial A} \approx \frac{R_{0}}{2} \sum_{i=0}^{N}\left(\frac{\omega_{i}}{\omega_{0}}\right)^{2} \mathbf{H}_{i} \cdot \mathbf{H}_{i}^{*},
$$

with $\omega_{i}$ as the circular frequency of the $i$ th mode. This approximation is backed up by several $R_{\mathrm{BCS}}$ calculations also showing the quadratic dependency on the frequency within a temperature range below the critical temperature.

We calculate the fundamental mode by frequency domain simulations with the power coupler as excitation source in order to incorporate potential coupling effects between the power and HOM coupler, which face each other on one side of the cavity. As the cavity provides one HOM coupler on each end, the location of the considered HOM coupler is specified in the following by either "input coupler side" or "tuner side" (Fig. 13). 
TABLE IV. Field and power parameters of the HOM couplers with respect to the fundamental mode.

\begin{tabular}{|c|c|c|c|c|c|c|c|c|c|c|}
\hline \multirow[b]{2}{*}{ Design } & \multicolumn{5}{|c|}{ HOM Coupler 1 (on tuner side) } & \multicolumn{5}{|c|}{ HOM Coupler 2 (facing the input coupler) } \\
\hline & $\begin{array}{c}|\mathbf{E}|_{\max } \\
{[\mathrm{MV} / \mathrm{m}]}\end{array}$ & $\begin{array}{c}|\mathbf{E}|_{\text {Notch }} \\
{[\mathrm{MV} / \mathrm{m}]}\end{array}$ & $\begin{array}{c}|\mathbf{H}|_{\max } \\
{[\mathrm{mT}]}\end{array}$ & $\begin{array}{l}I_{0}{ }^{\mathrm{a}} \\
{[\mathrm{A}]}\end{array}$ & $\begin{array}{c}P_{\text {extracted }}^{\mathrm{a}} \\
{[\mathrm{mW}]}\end{array}$ & $\begin{array}{c}|\mathbf{E}|_{\max } \\
{[\mathrm{MV} / \mathrm{m}]}\end{array}$ & $\begin{array}{c}|\mathbf{E}|_{\text {Notch }} \\
{[\mathrm{MV} / \mathrm{m}]}\end{array}$ & $\begin{array}{c}|\mathbf{H}|_{\max } \\
{[\mathrm{mT}]}\end{array}$ & $\begin{array}{l}I_{0}{ }^{\mathrm{a}} \\
{[\mathrm{A}]}\end{array}$ & $\begin{array}{c}P_{\text {extracted }}^{\mathrm{a}} \\
{[\mathrm{mW}]}\end{array}$ \\
\hline PROBE V1 & 13.29 & 5.82 & 10.19 & 69.25 & 14.907 & 3.36 & 2.15 & 3.56 & 24.82 & 0.962 \\
\hline PROBE V2 & 18.64 & 7.63 & 14.04 & 87.88 & 0.494 & 4.56 & 2.83 & 4.59 & 31.56 & 0.043 \\
\hline PROBE V3 & 18.15 & 7.87 & 14.42 & 94.24 & 0.045 & 4.58 & 2.92 & 5.23 & 33.80 & 0.011 \\
\hline HOOK V1 & 9.65 & 6.04 & 11.90 & 69.79 & 1.367 & 4.51 & 2.91 & 5.92 & 34.94 & 6.889 \\
\hline HOOK V2 & 9.64 & 5.98 & 11.91 & 70.49 & 4.412 & 4.51 & 2.88 & 6.03 & 35.29 & 0.455 \\
\hline HOOK V3 & 9.54 & 7.25 & 12.32 & 71.81 & 0.210 & 4.53 & 3.50 & 6.30 & 35.99 & 0.108 \\
\hline TESLA & 16.37 & 4.83 & 12.68 & 64.32 & 3.427 & 8.21 & 2.35 & 6.28 & 31.00 & 0.766 \\
\hline
\end{tabular}

${ }^{\mathrm{a}}$ with respect to a duty cycle of $10 \%$.

The field of the fundamental mode is mainly concentrated at the hook or probe antenna penetrating the cutoff tube (Fig. 14). The peak field values are summarized in Table IV. For the two coupler positions they range between 4 to $15 \mathrm{mT}$ or 3 to $18 \mathrm{MV} / \mathrm{m}$, respectively. The field in the gap of the (primary) notch filter is about 30\%-50\% lower than the maximum values on the antennas/ loops. The surface current and with it the dissipated power vary between the designs by $30 \%$. Here, the hook designs have an advantage when compared to the probe designs.

Note, the surface currents are significantly higher throughout for the HOM coupler on the tuner side. This is due to the fact that this coupler is located almost ten millimeters closer to the cavity than the coupler on the input coupler side. The latter could be inserted a few millimeters further into the cutoff tube but its performance would still not reach the level of the coupler on the tuner side. This is a drawback of the current cavity design and should be taken into account for future designs. In the following, we exclude the coupler on the input coupler side from our considerations since it is generally more weakly loaded.

In order to scale HOMs we may use the spectrum of the loss factor, $k_{\|}$, obtained by a wakefield simulation. Together with the specified peak beam current, $I_{b}$, and the bunch charge, $q_{b}$, the total HOM power for a SPL cavity yields:

$P_{\mathrm{tot}}^{\mathrm{HOM}}=k_{\|} q_{b} I_{b}=2.1 \mathrm{~V} / \mathrm{pC} \cdot 0.5 \mathrm{pC} \cdot 64 \mathrm{~mA}=67 \mathrm{~mW}$

This result confirms what was already concluded in [5] by beam dynamic simulations: HOMs are not critical for a stable operation of the SPL cavities as long as they are sufficiently far away from machine lines. However, as mentioned in the introduction, the machine lines depend also on the chopping pattern and may appear at any frequency. Therefore, it is difficult to predict the actual HOM power which may vary in a wide range even between the fabricated cavities (see frequency spread in Fig. 4). Based on the studies that have been done in [5] and the size limitation (45 $\mathrm{mm}$ HOM port diameter) it was decided to define the maximum feasible extracted HOM power per coupler to $100 \mathrm{~W}$. This restriction allows us to scale the HOMs assuming the worst case scenario.

The field distributions shown in Figure 15 are scaled to $1 \mathrm{~W}$ output power and may easily be rescaled. It also illustrates which modes couple well and therefore dissipate less power on the coupler surface for a given extracted power. In Figure 16, the resulting surface currents are compared between the considered HOMs. Since the hook designs as well as the PROBE V3 coupler offer a better transmission of the first dipole modes, these modes create considerably lower surface currents with respect to the extracted power.

Even if in most cases the surface currents are higher for the fundamental mode (Table IV) than for the HOMs (Fig. 16, assuming an overall extracted HOM power of $100 \mathrm{~W}$ ), one cannot conclude that this mode dominates the dissipated power because of the quadratic rise of the surface resistance with the frequency. Thus for the $\mathrm{TM}_{011}$ mode at $1.33 \mathrm{GHz}$, the resistance is around four times higher than for the fundamental mode at $704.4 \mathrm{MHz}$ and for the $\mathrm{TM}_{021}$ at $2.086 \mathrm{GHz}$ it rises by almost one order of magnitude. From (2) and (3) we derive a relation between the contributed dissipated power originated from the HOMs and from the fundamental mode on the coupler surface according to:

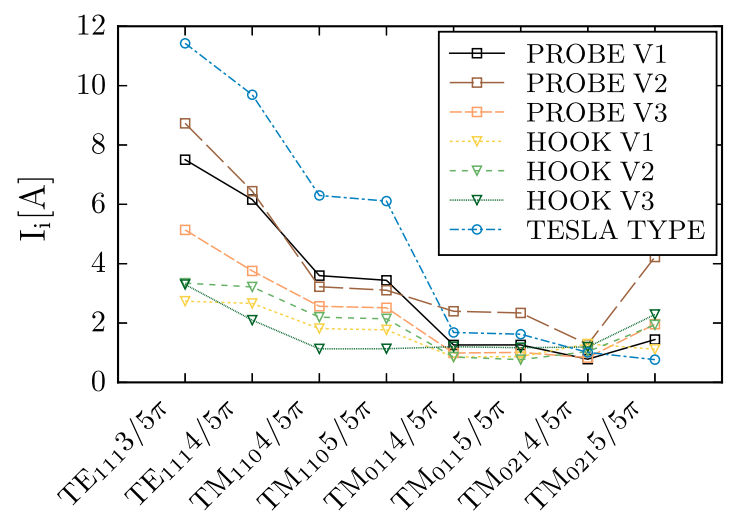

FIG. 16. Surface currents $I_{\text {surf }}$ of the HOM coupler on the tuner side for different modes calculated via (2). Each of the HOMs are normalized to an extracted power of $1 \mathrm{~W}$. 


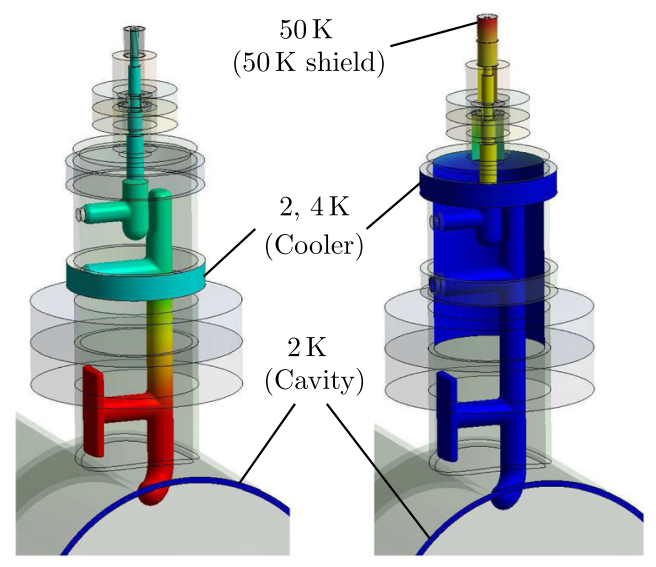

FIG. 17. Boundary conditions for the steady state thermal simulations using ANSYS. Left: Power dissipation caused by the electromagnetic fields in the coupler (mode heating). Right: Heat load coming from the rf cable.

$$
\frac{P_{\text {dissipated }}^{\mathrm{HOM}}}{P_{\text {dissipated }}^{\text {fundamental }}}=\frac{1}{I_{0}^{2}} \sum_{i=1}^{N}\left(\frac{\omega_{i}}{\omega_{0}}\right)^{2} I_{i}^{2} \text {. }
$$

If we weigh all considered HOMs by their $(R / Q)$-values and assume an accumulated output power of $100 \mathrm{~W}$, the fundamental mode dominates the total dissipated power with more than $60 \%$. The only exception is the TESLA design where the HOMs dominate with $65 \%$.

\section{Thermal simulation}

In the following, the thermal analysis is presented for the PROBE V3 coupler where the fundamental mode takes around $75 \%$ of the total dissipated power. Based on the scaled magnetic fields we are able to create a composed heat flow serving as boundary condition for our steady state thermal analysis (ANSYS). Furthermore the cavity wall is assumed to be at a fixed temperature of $2 \mathrm{~K}$ (Fig. 17 left). The temperature dependent materials considered for the thermal simulation are summarized in Table V. The thermal conductivity of niobium significantly depends on its residual resistance ratio. Before manufacturing the coupler,

TABLE V. Materials for the thermal simulations.

\begin{tabular}{llc}
\hline \hline Component & \multicolumn{1}{c}{ Material } & Ref. \\
\hline $\begin{array}{l}\text { Cavity and beam } \\
\text { pipe section }\end{array}$ & $\begin{array}{c}\text { Niobium with } \\
\text { RRR }=60,380\end{array}$ & {$[3,25]$} \\
HOM coupler & $\begin{array}{l}\text { Niobium with } \\
\text { RRR }=60,380\end{array}$ & {$[3,25]$} \\
Coupler tube & Niobium with & {$[3,25]$} \\
& RRR $=60,380$ & \\
Flanges & Stainless steel & {$[25]$} \\
Ceramic window & Sapphire & {$[25]$} \\
Conductors of the rf cable & Copper with RRR $=60$ & {$[25]$} \\
Insulator of the rf cable & Polyethylene & {$[25]$} \\
\hline \hline
\end{tabular}

the pure material will have a RRR of 300. During the fabrication, this value will change only marginally. For comparison, we consider the values 60 and 380 in the simulations.

The temperature dependency of the surface resistance yields an implicit formulation of the steady state thermal problem. The thermal simulation provides the peak temperature on the coupler surface as a function of the reference surface resistance $R_{0}$ and the residual resistance ratio of niobium:

$$
T_{\max }=\max _{\mathbf{r} \in \partial \mathbf{\Omega}_{\text {coupler }}}\left\{\mathrm{T}\left(\mathbf{r}, R_{0}, \mathrm{RRR}\right)\right\} .
$$

The reference surface resistance again is a function of the temperature and in the worst case may be expressed as:

$$
R_{0}=R_{\mathrm{BCS}}\left(T_{\max }, \mathrm{RRR}, f_{0}\right),
$$

with $f_{0}=704.4 \mathrm{MHz}$. This conservative approach assumes a homogeneously distributed surface resistance determined by the maximum temperature on the coupler surface. Instead of solving the implicit problem (5) directly within ANSYS, we use a graphical approach that allows to discuss quantitatively and qualitatively not only the temperature range but also the range of the reference surface resistance in which the coupler operates stably.

Figure 18 shows the results of the thermal simulations for both the low and high RRR values. It depicts the simulated maximum temperature on the coupler surface as a function of the reference surface resistance $R_{0}$ according to (5a) (solid curves). Besides the pure coupler model we consider two cases in which the coupler is cooled by thermal conductivity at 2 and $4 \mathrm{~K}$ as shown in Fig. 17. In order to find the solutions which fulfill (5b), the inverse function of the BCS surface resistance is shown in dashed black in Fig. 18 for three different frequencies between 0.7 and $2.1 \mathrm{GHz}$. It would be sufficient to only consider $R_{\mathrm{BCS}}$ at $f_{0}=704.4 \mathrm{MHz}$ as claimed by (5b), but considering different frequencies introduces a certain fuzziness (highlighted in gray). Instead of a precise value, we determine a range for the maximum temperature on the coupler surface that may be interpreted with a standard deviation or accuracy. The interceptions of the solid curves in Fig. 18 with the gray zones yield a thermal equilibrium. This is also true for any other heat source, such as the heat transfer via the output port discussed in the next section.

If due to any transitional effect (e.g. pressure fluctuations) a nonequilibrium state above the gray zone is taken, then the temperature would rise until entering in an equilibrium state. On the other hand, the temperature would decrease if a state below the gray zone is taken. For example in Fig. 18(a), the coupler will never have a stable temperature below $4.2 \mathrm{~K}$ if a $4 \mathrm{~K}$ thermalization is applied. With a $2 \mathrm{~K}$ cooling contact or even without additional cooling the temperature could always return to close to $2 \mathrm{~K}$ as long as $R_{0}<5 \mu \Omega$. Indeed, the cooling 


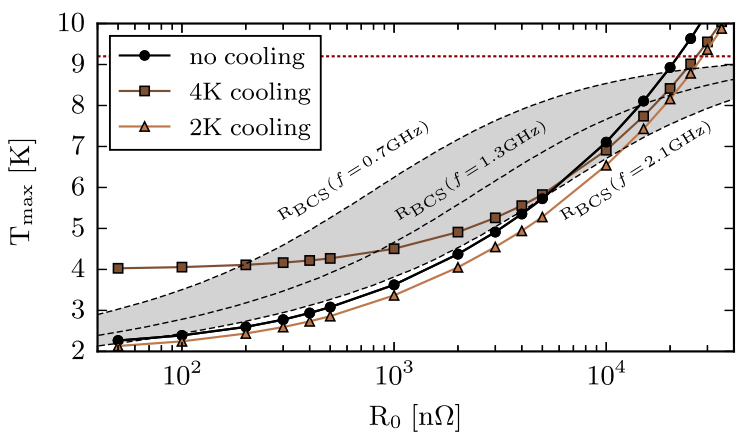

(a)

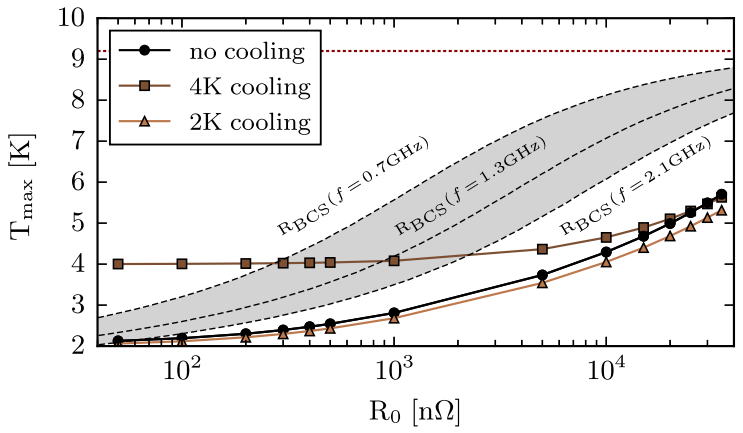

(b)

FIG. 18. Maximum temperature on the PROBE V3 surface versus the surface resistance with the thermal boundary conditions described in the text. The critical temperature of $9.2 \mathrm{~K}$ is highlighted in red. For comparison, the BCS resistance computed by [26] is shown in dashed black for different frequencies. A thermal equilibrium may only exist within the gray highlighted zones. (a) $\mathrm{RRR}=60$. (b) $\mathrm{RRR}=380$.

mechanism acts only at higher temperatures with $T>6 \mathrm{~K}$. Furthermore, a thermalization at $4 \mathrm{~K}$ seems inefficient. This is true also for higher RRR [Fig. 18(b)].

For RRR $=60$ [Fig. 18(a)], the heat cannot be evacuated anymore if the temperature rises above $8.8 \mathrm{~K}$ at surface resistances of $R_{0}>20 \mu \Omega$ and the coupler quenches. However, it might work up to a temperature of $8 \pm$ $0.5 \mathrm{~K}$ and $R_{0} \leq 10 \mu \Omega$. A cooling plate at $2 \mathrm{~K}$ may allow half a degree more and twice the surface resistance. But the more important result is that a surface resistance in this order is very high and unlikely, which means the coupler would generally operate at temperatures close to $2 \mathrm{~K}$ even without cooling mechanisms.

For RRR $=380$ [Fig. 18(b)], the situation becomes less critical due to the higher thermal conductivity. Here we may not even see any temperature increase above $2.1 \mathrm{~K}$ up to surface resistances of $0.1 \mathrm{~m} \Omega$.

To summarize this section, the heating of the HOM coupler by the electromagnetic fields appears to be minor unless the coupler is not well surface treated. A residual resistance ratio around 300 is realistic. The described analysis applied to the remaining couplers leads to very similar results with slightly better behavior for the hook couplers because their fixing at the tube wall is lower (Fig. 6).

\section{B. Heating from the outside}

Another heat source is the rf cable as it constitutes a thermal bridge between the $50 \mathrm{~K}$ shield and the output port of the HOM coupler [27]. In this case, a pure steady state thermal analysis is carried out without taking into account electromagnetic fields. For this purpose, we consider the cavity at a temperature of $2 \mathrm{~K}$ and the output port extended by a length $l_{\mathrm{RF}}$ cable with a constant temperature of $50 \mathrm{~K}$ at the end (Fig. 17 right).

In Fig. 19, the maximum temperature and heat load as functions of the cable length are shown. We assumed different radii for the inner conductor $(1.00,1.25,1.50$, $1.75 \mathrm{~mm}$ ) and a thickness of $0.25 \mathrm{~mm}$ for the outer conductor. For the considered cable parameters, the temperature easily exceeds the critical temperature of niobium. This is also the case for a relatively long distance to the $50 \mathrm{~K}$ shield and hence requires a cooling of the coupler tube and of the cable by heat intercepts.

In Fig. 20, the modeled rf cable is assumed $500 \mathrm{~mm}$ long with a $2 \mathrm{~mm}$ thick inner conductor. Furthermore, a heat sink around the coupler tube is applied with a given contact temperature $T_{\text {contact }}$. The coupler temperature and the heat load are considered as functions of the contact temperature.
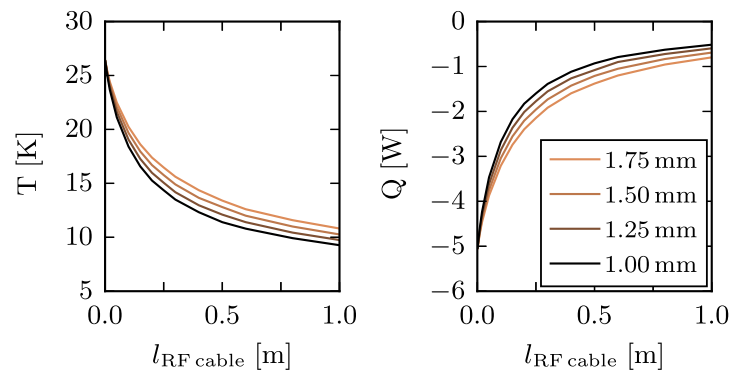

FIG. 19. Left: Maximum temperature on the coupler surface for different radii of the inner conductor of the rf cable as a function of the cable length (distance to the $50 \mathrm{~K}$ shield). The heat source is the $50 \mathrm{~K}$ shield itself and the heat sink is the cavity wall at $2 \mathrm{~K}$. The values are consistently above the critical temperature of $9.2 \mathrm{~K}$. Right: the corresponding heat.

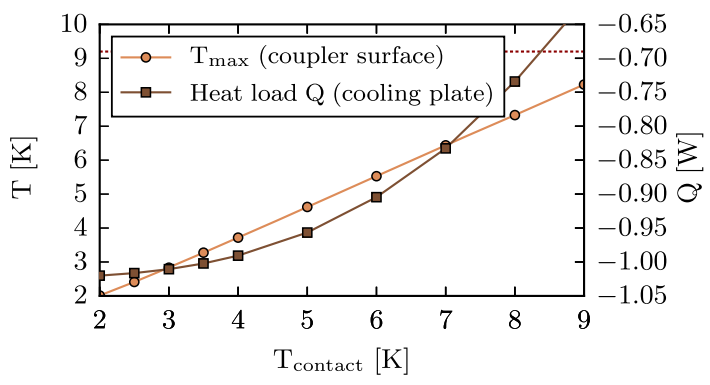

FIG. 20. Maximum temperature on the coupler surface for different contact temperatures of the cooling heat sink around the coupler tube. The heat source is the $50 \mathrm{~K}$ shield, the heat sink is the cavity wall at $2 \mathrm{~K}$ and the cooler in contact with the coupler tube. The critical temperature of $9.2 \mathrm{~K}$ is highlighted. 
Besides the resulting peak values, the graph shows the heat load which has to be established by the heat sink to achieve the stated temperature values. In order to keep the temperature-dependent surface resistance as low as possible, a heat load $\geq 950 \mathrm{~mW}$ should be considered. In contrast the maximum power consumption for one HOM coupler should be $\leq 1 \mathrm{~W}$. In the current design of the SPL cryostat, a cable length of $2 \mathrm{~m}$ is foreseen. Furthermore two heat intercepts are used along the cable to cool the outer conductor which reduces the heat transfer to less than $500 \mathrm{~mW}$ and provides enough margin.

\section{MULTIPACTING}

Multipacting is an avalanche-like growth of free electrons [28] that frequently occurs during the conditioning of $\mathrm{rf}$ cavities and couplers. It appears if the rf field drives electrons on repetitive trajectories providing a continuous impact on the wall and if impact energy and material specific electron yield (SEY) favor the emission of secondary electrons. Latter requires SEY $>1$ at the impact energy (Fig. 21). As a result, the temperature rapidly rises at the affected locations. Multipacting can considerably extend the processing time of a rf-device (soft barriers) or even limit its performance (hard barrier). Though it is a very serious obstacle for operation, we consider it at the very end of the design phase because a small change in the coupler geometry can have a major impact. Two independent programs have been used, both combining 3D electromagnetic field simulations with particle tracking: ACE3P (ADVANCED COMPUTATIONAL ELECTROMAGNETICS 3D PARALLEL) and CST MWS together with Particle Studio (PS). Details can be found in [29-32].

The model consists of the cavity and one HOM coupler installed on the tuner side. This means that possible multipacting barriers presented in this section would appear at slightly higher field levels if the coupler is installed on the other cutoff tube of the cavity. Furthermore, we restricted our investigations to the PROBE V3 and HOOK V3 coupler and eventual modifications. For comparison, we applied two different material properties shown in Fig. 21 with SEY peak values of 2.1 (in the following referred to as copper) and 1.5 (referred to as niobium).

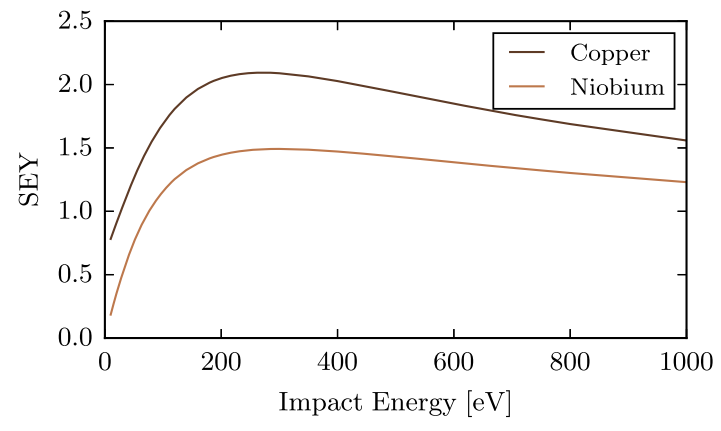

FIG. 21. Secondary electron yield for copper and niobium (after $300{ }^{\circ} \mathrm{C}$ bake) [12].
The analysis in both codes is very similar: An eigenmode simulation carried out by either OMEGA3P (part of ACE3P) or CST MWS provides the rf field of the fundamental mode inside the HOM coupler which is subsequently used in the particular tracking code (TRACK3P or CST PS) to identify resonant trajectories and multipacting barriers. The latter is computationally more expensive for complete analyses of a rf device when more particles are tracked. Therefore, the initial particle emission from the entire coupler surface was subdivided into a total of 11 regions, hence, 11 separate simulations. Each simulation incorporates three field scans covering a gradient range of $5 \mathrm{kV} / \mathrm{m}$ to $30 \mathrm{MV} / \mathrm{m}$. Most results presented here are obtained by ACE3P which allows detailed and relatively fast analysis using high-performance parallel computing. CST was used to verify particular multipacting barriers and to gradually reduce and suppress them by design modifications.

Both, the PROBE V3 and HOOK V3 coupler reveal qualitatively the same multipacting characteristics due to their similarities in the notch and HOM filter. Resonant trajectories were found at three regions which will be discussed separately in the following. With sufficiently high SEY, electrons on these trajectories result in two-point first order multipacting.

\section{A. Notch filter}

First, the original PROBE V3 coupler was analyzed showing resonant trajectories between $0.1-0.35 \mathrm{MV} / \mathrm{m}$ (Model 1 in Figs. 22, 23 and 24). These correspond to a soft multipacting barrier because the trajectories are not stable and the particles gradually move out of the gap. Using the SEY of niobium (Fig. 21), the averaged secondary electron yield $\langle$ SEY $\rangle$ after $50 \mathrm{rf}$ cycles is far below 1 because of impact energies that are too low $(<60 \mathrm{eV})$. This means that, on average, no secondary electrons are excited. Instead, primary electrons will be absorbed. In case of copper, the impact energy suffices to emit secondary electrons and result in a maximum $\langle\mathrm{SEY}\rangle$ of 1.25. However this averaged secondary electron yield is measured over the life time of a particle which does not

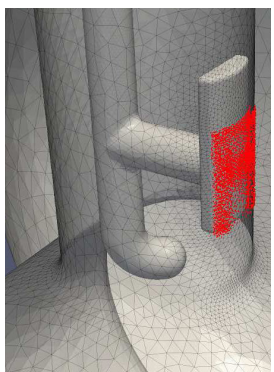

(a) Model 1

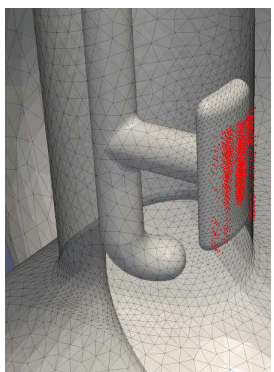

(b) Model 2

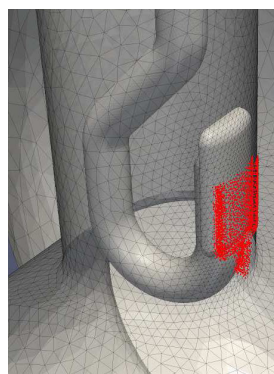

(c) Model 3
FIG. 22. Resonant trajectories between the tube and the lower part of the notch filter plate of (a) original PROBE V3, (b) modified PROBE V3, and (c) modified HOOK V3 coupler. 


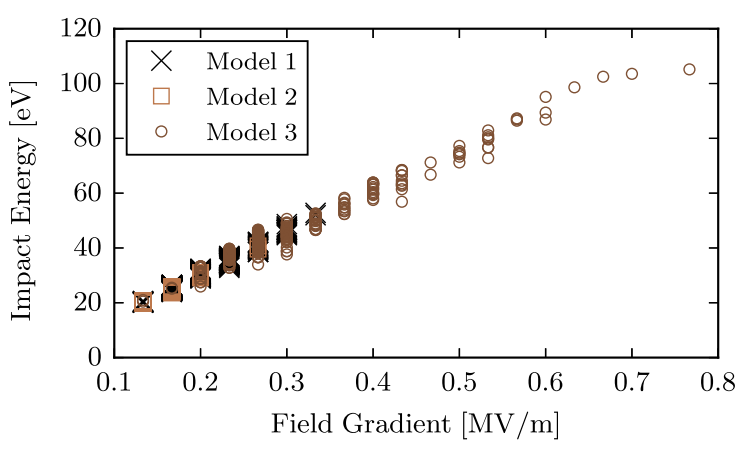

(a)

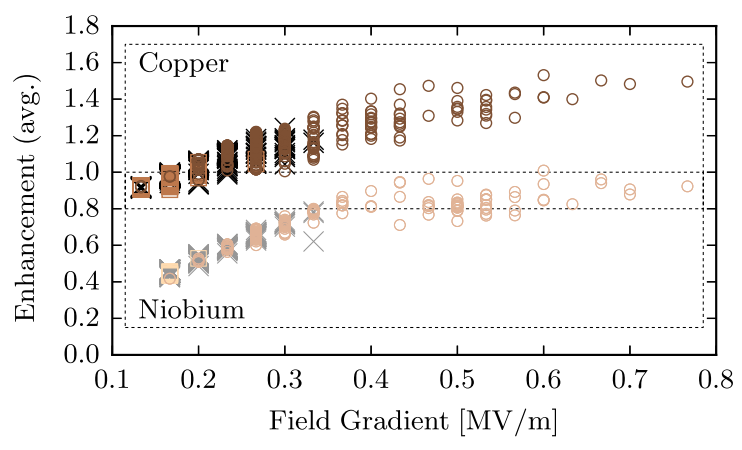

(b)

FIG. 23. Impact energy and averaged secondary electron yield of resonant trajectories at the notch filter for the models shown in Figure 22. Below the darker colors are related to copper while the lighter colors are related to niobium.

necessarily guarantee that multipacting occurs. The particle evaluation simulated with the particle-in-cell solver from CST shows a saturation after 20 rf cycles and a subsequent decrease of the particle number (Figure 24).

Though multipacting seems not to be an issue at the notch filter we increased the roundings at the edges to allow the electrons to escape the gap more quickly. This is referred to as Model 2 in Fig. 22. The energy range of the resonant trajectories is reduced, with a corresponding reduction in the $\langle\mathrm{SEY}\rangle$ from 1.25 to 1.1 , as shown in Fig. 23. The effect of this modification can be best seen

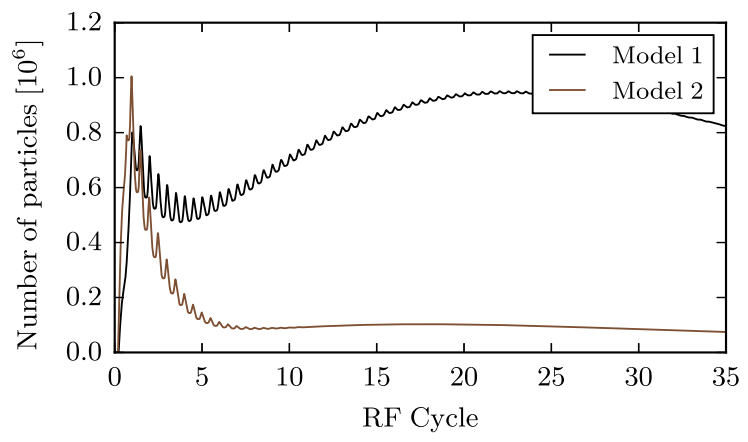

FIG. 24. Particle evaluation at $0.25 \mathrm{MV} / \mathrm{m}$ for the first two models in Fig. 23 simulated with CST PS. Initial electrons are directly emitted from the notch plates. from the particle evaluation simulated with CST (Fig. 24) revealing an instant decrease of the electron number.

The same modification was applied to the HOOK V3 coupler before analyzing its multipacting characteristics [Fig. 22(c)]. The barrier covers a larger field range between 0.1 to $0.8 \mathrm{MV} / \mathrm{m}$ (Fig. 23). This is caused by resonant trajectories between the hook and tube directly below the notch plate. The averaged secondary electron yield is higher than for the previously discussed models but in the case of niobium this is still below one.

\section{B. HOM passband filter}

A hard multipacting barrier was found in the capacitor gap of the passband filter ( $\mathrm{C} 3$ in Fig. 8) for the original PROBE V3 coupler which is referred to as Model 1 in Figs. 25 and 26.

The potential of multipacting is also at a field level of 14 to $17 \mathrm{MV} / \mathrm{m}$. Hence, the conditioning of the cavity or in the worst case its operation and performance are most likely influenced by this barrier. For this reason, we changed one of the capacitor plates from the original concave profile to a flat profile and then to a convex one (Fig. 25). In the latter case the barrier is significantly reduced down to a range of 3 to $5 \mathrm{MV} / \mathrm{m}$ with a $\langle\mathrm{SEY}\rangle<0.9$ for niobium (Model 3 in Figure 26). Basically, the same modification with slightly different dimensions was applied on the HOOK V3 coupler to likewise suppress multipacting at the HOM passband filter.

The vertically oriented capacitor ( $\mathrm{C} 4$ in Fig. 8) is not affected by multipacting, however it was modified as shown in Figure 25 because of mechanical reasons (larger gap size requires larger surface).

\section{Antenna}

Both the PROBE V3 and the HOOK V3 coupler depict resonant trajectories at the rear side of their antennas (Fig. 27). Due to the large distance to the tube wall, this behavior appears at relatively high field gradients starting from $5 \mathrm{MV} / \mathrm{m}$ (Fig. 28). In spite of a high $\langle\mathrm{SEY}\rangle$, most of the corresponding particles show a very short life time of

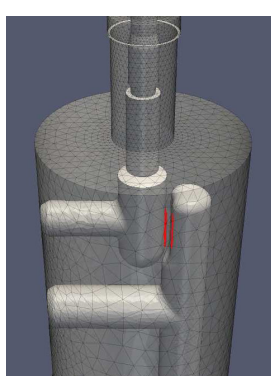

(a) Model 1

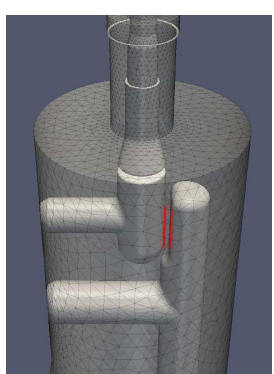

(b) Model 2

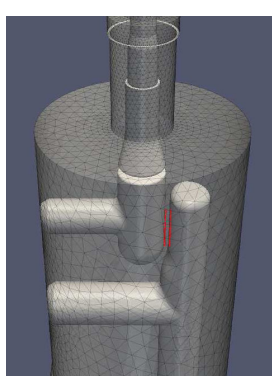

(c) Model 3
FIG. 25. Resonant trajectories at the HOM passband filter of the PROBE V3 coupler for (a) the original concave gap, (b) the flat gap, and (c) convex gap. 


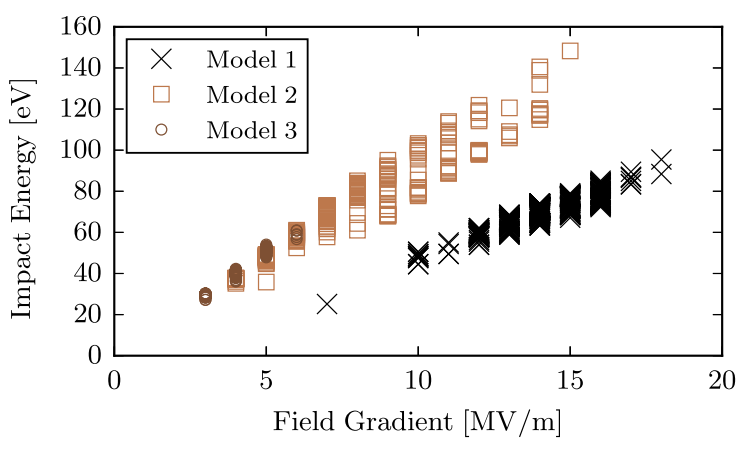

(a)

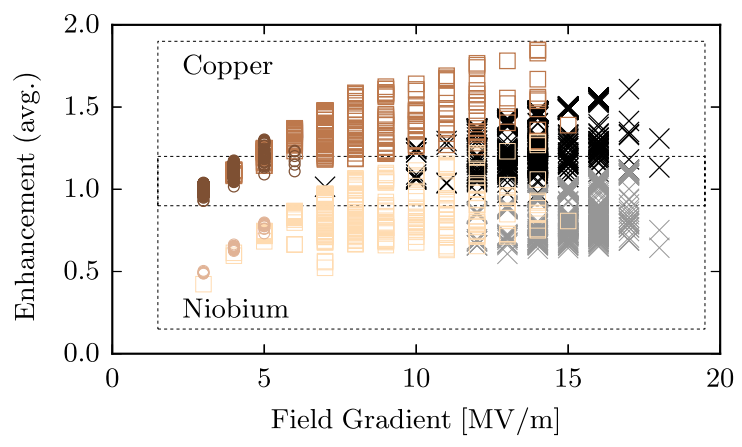

(b)

FIG. 26. Impact energy and averaged secondary electron yield of resonant trajectories at the HOM passband filter for the models shown in Fig. 25. Below the darker colors are related to copper while the lighter colors are related to niobium.

less than $15 \mathrm{rf}$ cycles, hence no electron growth in the long term is possible. There is only one exception for the HOOK V3 coupler at around $30 \mathrm{MV} / \mathrm{m}$. These trajectories are stable revealing a hard multipacting barrier. However, as the cavity is not supposed to operate at this field gradient, we retain the present design.

Coupler orientations different from the one shown in Figure 27 have been analyzed for the PROBE V3 coupler with qualitatively the same results: unstable trajectories. For the HOOK V3 coupler, this test was not necessary because any other orientation increases the coupling to the fundamental mode.

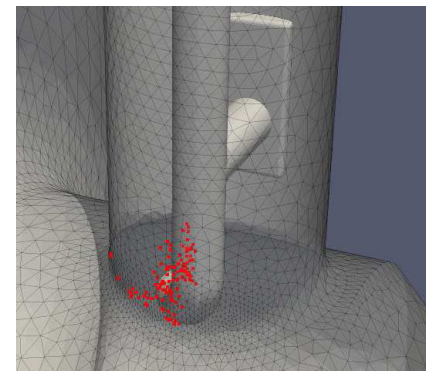

(a) Model 1

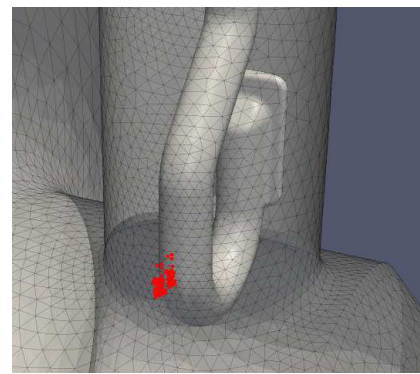

(b) Model 2
FIG. 27. Resonant trajectories at the probe and hook antenna. (a) PROBE V3. (b) HOOK V3.

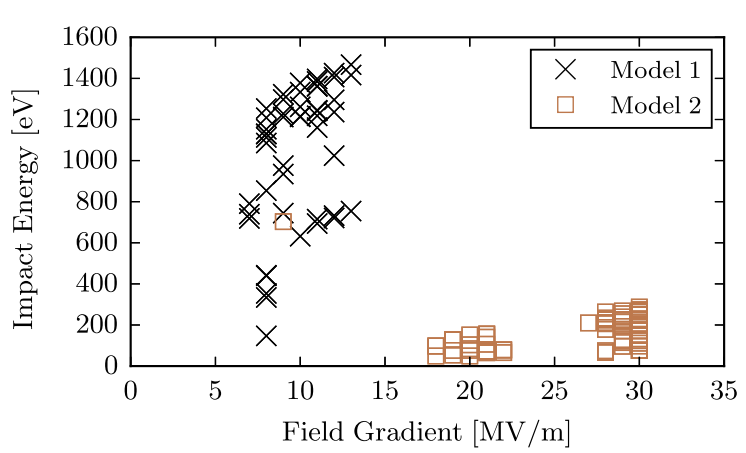

(a)

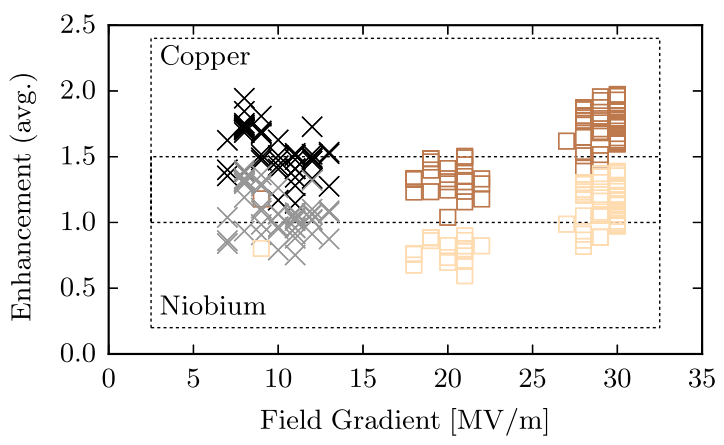

(b)

FIG. 28. Impact energy and averaged secondary electron yield of resonant trajectories at the probe or hook antenna (refer Fig. 27). Below the darker colors are related to copper while the lighter colors are related to niobium.

\section{3D-PRINTED HOM COUPLER PROTOTYPE}

Based on rf and thermal simulations for the designs in an earlier stage [33], it was decided to fabricate a HOM coupler with probe antenna, basically a previous version of the PROBE V3 design. This coupler type was selected for the following features: (i) Notch filter with a relatively high bandwidth $(140 \mathrm{MHz}$ at $-100 \mathrm{~dB})$ and therefore very robust. (ii) High selectivity, meaning steep transition between stop band (notch filter) and passband. (iii) Best coupling to the monopole HOMs at $1.33 \mathrm{GHz}$. (iv) No active cooling of the antenna necessary (see Section IVA). The differences to the design shown in Figure 6 are minor and their effect on the rf characteristics is negligible. The newer version is slightly easier to fabricate. The prototype discussed in this paper is a 3D-printed model in order to accelerate the whole design process and to verify the transmission behavior. The rapid prototype is made of plastic and coated with copper (Fig. 29).

A disadvantage of this prototype is the relatively high surface roughness of $R_{t}=240 \mu \mathrm{m}^{2}$ Nevertheless, after tuning the notch filter, the prototype has the simulated transmission behavior as presented in the following. Furthermore, we present several $Q_{\text {ext }}$ analyses and compare

\footnotetext{
${ }^{2}$ Maximum height of the profile.
} 


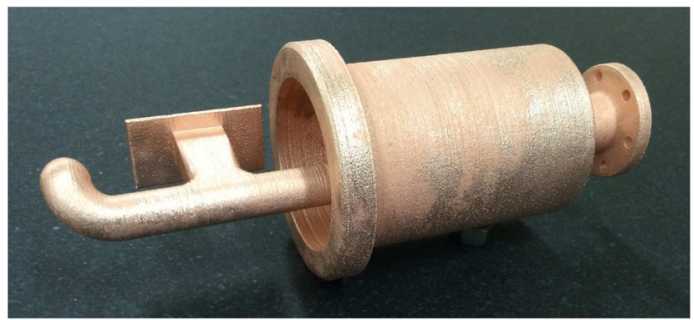

FIG. 29. The 3D printed HOM coupler. The prototype is made of plastic and covered by a copper layer of approximately $0.1 \mathrm{~mm}$ thickness (PROBE V3).

them with simulations. All described measurements have been carried out with a copper SPL cavity at low power and at room temperature (Fig. 30).

\section{A. $S_{21}$ measurements}

By default the coupler is adjusted as shown in Fig. 30 with a penetration depth of $11.5 \mathrm{~mm}$ and the capacitive plate facing the viewer, which means that the plate is perpendicular to the beam pipe axis as depicted in Fig. 1(a). A rotatable flange allows a continuous variation of the antenna orientation. Furthermore, the plastic flange allows off-axis movement of the HOM coupler to change the gap distance of the (primary) notch filter. The passband is tuned by adjusting the distance of the feed-through to the coupler (C4 in Fig. 8). This distance affects especially the transmission for the first dipole band at around $900 \mathrm{MHz}$ as well as for the second monopole band around $1.33 \mathrm{GHz}$. The coupler was tuned to get as close as possible to the simulated results.

Figure 31 shows the transmission measured from a reference antenna at the beam port to the output port of the HOM coupler. The setup is exactly the same as shown in Fig. 30. The measurement agrees reasonably well with the desired transmission in Fig. 10 for the PROBE V3 coupler. For a better comparison, a frequency domain simulation of the entire setup is shown in Figure 31 as well.

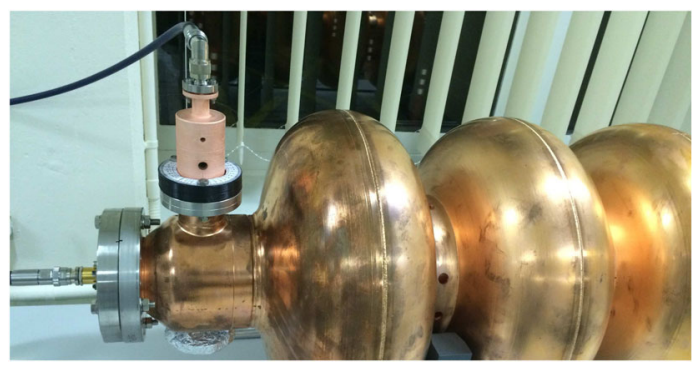

FIG. 30. HOM coupler prototype mounted on a copper SPL cavity with a rotatable flange. Here, the rf transmission of the coupler is measured using a reference antenna at the beam pipe port close to the HOM antenna. The input coupler used for measurements involving the cavity characteristics (e.g. $\left.Q_{\text {ext }}\right)$ is on the other side of the cavity (not shown).

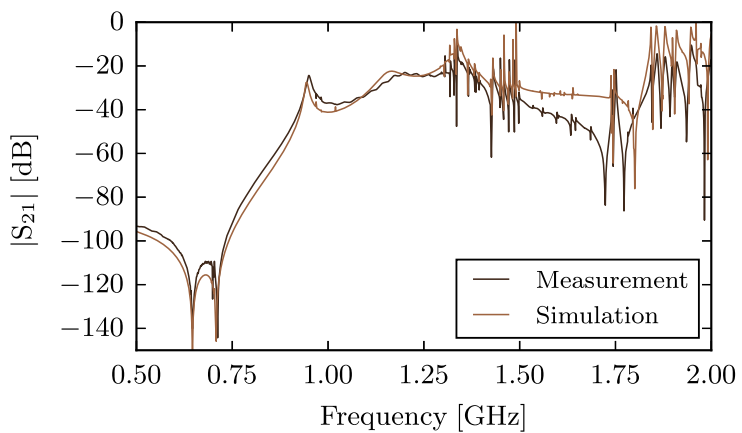

FIG. 31. $S_{21}$ Transmission from the beam pipe (using a probe antenna) to the HOM coupler port (Fig. 30).

The rejection of the fundamental mode is larger than $110 \mathrm{~dB}$. The transmission of modes in the second dipole band around $1 \mathrm{GHz}$ is relatively weak with less than $-35 \mathrm{~dB}$, in case of the prototype. This was to be expected as the probe designs are not optimal for the first dipole bands.

\section{B. $Q_{\text {ext }}$ measurements}

$Q_{\text {ext }}$ was measured for modes up to $2.1 \mathrm{GHz}$. The standard procedure $[9,10]$ is as follows: (i) Measuring $Q_{L}$ by $S_{21}$ (transmission measurement). (ii) Measuring the coupling factor $\left.\beta\right|_{\text {hom }}$ and $\left.\beta\right|_{\text {input }}$ for the antennas from $S_{11}$ and $S_{22}$, which is either the standing wave ratio (overcoupling) or its inverse (undercoupling). (iii) Determining $Q_{0}$ from $Q_{0}=Q_{L}\left(1+\left.\beta\right|_{\text {hom }}+\left.\beta\right|_{\text {input }}\right)$. (iv) Determining $Q_{\mathrm{ext}}$ from $Q_{\mathrm{ext}}=Q_{0} / \beta$. In case of a very low coupling, the error of the reflection type measurement based on $S_{11}$ or $S_{22}$ becomes significant, which appears easily at room temperature, i.e. $293 \mathrm{~K}$. As an example, consider a mode with $Q_{\text {ext }}>10^{6}$ which results in $\beta<0.01$ whereas it would be 5 orders of magnitude higher at $2 \mathrm{~K}$. A possible way to bypass this problem and to achieve reliable results for those coupling factors requires a stronger coupled reference antenna (or in our case the input antenna). If we consider a mode which couples very weakly to the HOM coupler antenna, for example the fundamental mode, its coupling factor would be calculated according to formula (6) rather than using the standing wave ratio as described before:

$$
\left.\beta\right|_{\text {hom }}=\frac{\left|S_{21}\right|^{2}}{\frac{\left.4 \beta\right|_{\text {input }}}{\left(\left.\beta\right|_{\text {input }}+1\right)^{2}}-\left|S_{21}\right|^{2}} .
$$

The results are shown in Fig. 32 and details for the interesting modes can be found in Table VI. In parts they deviate significantly from their simulated value. This might be due to cavity imperfection and the high surface roughness of the coupler. Moreover, the cavity was tuned several times and detuned in previous tests, yielding a variation in mode frequency.

Using only one coupler with a penetration depth of $11.5 \mathrm{~mm}$, the external quality factor for modes in the $\mathrm{TM}_{011}$ 
TABLE VI. Measured and simulated external $Q$ values for the most important modes, using one or two couplers respectively.

\begin{tabular}{|c|c|c|c|c|c|c|}
\hline \multirow[b]{2}{*}{ Mode Type } & \multicolumn{2}{|c|}{ Frequency $[\mathrm{MHz}]$} & \multicolumn{3}{|c|}{ External Q } & \multirow{2}{*}{$\begin{array}{c}R / Q[\Omega] \\
\text { Simulation }\end{array}$} \\
\hline & Measurement & Simulation & Measurement & Simulation & Simulation $^{\mathrm{a}}$ & \\
\hline $\mathrm{TM}_{010} 5 / 5 \pi$ & 703.34 & 704.38 & $4.4 \times 10^{14}$ & $1.9 \times 10^{15}$ & $9.6 \times 10^{16}$ & 566 \\
\hline \multirow{2}{*}{$\mathrm{TE}_{111} 3 / 5 \pi$} & 919.06 & 917.97 & $1.0 \times 10^{7}$ & $1.1 \times 10^{7}$ & $4.4 \times 10^{5}$ & 57 \\
\hline & 919.84 & 918.12 & $8.0 \times 10^{7}$ & $1.1 \times 10^{8}$ & $2.5 \times 10^{4}$ & \\
\hline \multirow[t]{2}{*}{$\mathrm{TE}_{111} 4 / 5 \pi$} & 942.97 & 942.87 & $8.3 \times 10^{4}$ & $7.4 \times 10^{4}$ & $6.5 \times 10^{4}$ & 60 \\
\hline & & 943.23 & & $1.7 \times 10^{6}$ & $1.8 \times 10^{4}$ & \\
\hline \multirow[t]{2}{*}{$\mathrm{TM}_{110} 3 / 5 \pi$} & 1014.3 & 1015.0 & $1.1 \times 10^{7}$ & $1.1 \times 10^{7}$ & $1.5 \times 10^{5}$ & 36 \\
\hline & & 1015.1 & & $1.1 \times 10^{9}$ & $4.1 \times 10^{6}$ & \\
\hline \multirow{2}{*}{$\mathrm{TM}_{110} 4 / 5 \pi$} & 1019.5 & 1020.7 & $2.2 \times 10^{7}$ & $2.3 \times 10^{7}$ & $8.6 \times 10^{6}$ & 25 \\
\hline & & 1020.7 & & $3.4 \times 10^{5}$ & $3.2 \times 10^{5}$ & \\
\hline $\mathrm{TM}_{011} 4 / 5 \pi$ & 1318.9 & 1317.4 & $1.5 \times 10^{5}$ & $1.2 \times 10^{5}$ & $1.1 \times 10^{4}$ & 39 \\
\hline $\mathrm{TM}_{011} 5 / 5 \pi$ & 1329.1 & 1329.2 & $1.8 \times 10^{5}$ & $1.9 \times 10^{5}$ & $6.4 \times 10^{3}$ & 140 \\
\hline $\mathrm{TM}_{021}$ & 2088.7 & 2089.4 & $1.7 \times 10^{6}$ & $8.5 \times 10^{5}$ & $3.7 \times 10^{4}$ & 21 \\
\hline
\end{tabular}

${ }^{\mathrm{a}}$ Setup with two HOM couplers simulated and $15 \mathrm{~mm}$ penetration depth.

are close to $10^{5}$. For the measured $\mathrm{TM}_{022}$ mode this value is increased by one order of magnitude and even more for some of the considered dipole modes (depending on their polarization). With a penetration depth of $15 \mathrm{~mm}$ and an additional HOM coupler on the input coupler side, $Q_{\text {ext }}$ would be below the specified limit of $10^{5}$ for all regarded HOMs, according to simulations.

The different polarizations of dipole modes may vary in a wide range concerning their $Q_{\text {ext }}$ as it is depicted in Fig. 32 (only for the $\mathrm{TE}_{111}$ band). However, only in a few cases were we able to successfully measure them separately using vector fitting methods to extract their quality factors [34].

Moreover, we investigated the impact of the coupler orientation on $Q_{\text {ext }}$. Here, the orientation is related to the azimuthal angle of the HOM coupler which is changeable due to the rotatable flange. We define the default position with an angle of $0^{\circ}$ (notch filter plate perpendicular to the beam axis). In case of $-90^{\circ}$, the capacitive plate of the

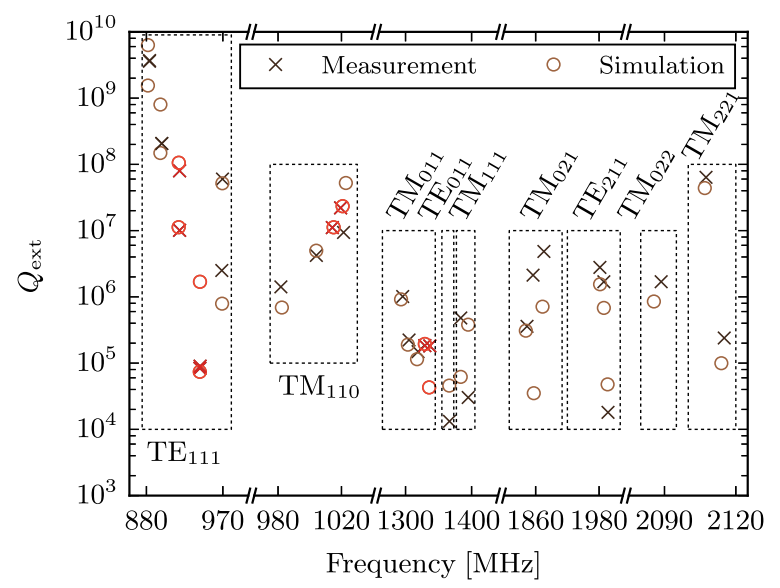

FIG. 32. Measured and simulated external $Q$ for the HOMs of several bands considering one coupler on the tuner side with a penetration depth of $11.5 \mathrm{~mm}$. Modes with an $(R / Q)$ larger than $10 \mathrm{Ohm}$ are highlighted in red. (primary) notch filter faces the cavity and for $+90^{\circ}$ it faces the beam pipe port. For each angle the notch filter has to be readjusted to the right frequency such that the gap distance varies with the angle in a range of $\pm 0.25 \mathrm{~mm}$. This complicates the simulations and causes the deviation from the measured data shown in Fig. 33 for modes in the first dipole band. Measured and simulated results agree well for

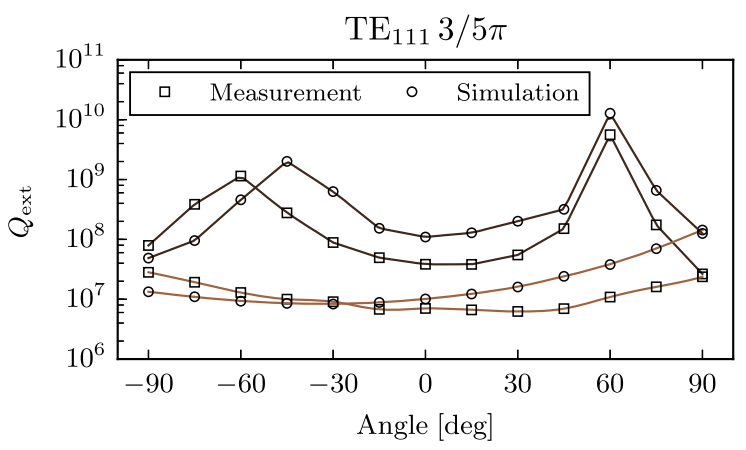

(a)

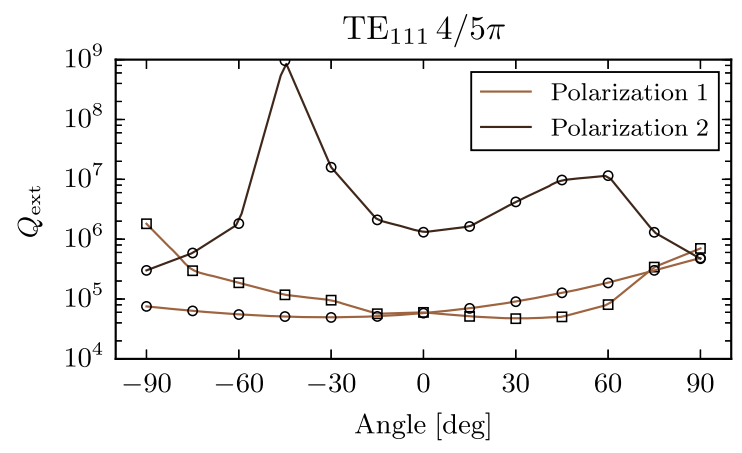

(b)

FIG. 33. Simulated and measured $Q_{\text {ext }}$ as a function of the azimuthal angle of the HOM coupler for two dipole modes and their polarizations in the $\mathrm{TE}_{111}$ band. Below, it was not possible to measure $Q_{\text {ext }}$ for both polarizations. 
the $\mathrm{TE}_{111} 3 / 5 \pi$ mode but for the $\mathrm{TE}_{111} 4 / 5 \pi$, it was not possible to differentiate between the two polarizations.

This dependency has also been measured for other modes in order to find an optimal angle. Based on the measurements and simulations, an angle of $30^{\circ}$ seems to be a good compromise.

\section{CONCLUSIONS}

In this paper, we have presented a detailed analysis of HOM couplers foreseen for the high-beta SPL cavities. Several measurements have been performed on copper and niobium cavities in order to characterize and compare their HOM spectra. The relatively low variations of mode frequencies as well as $Q$ factors relax the requirement for the filter passband.

Seven design approaches of HOM couplers have been presented, which differ in the kind of field coupling, notch filter and selectivity, passband characteristic (preferred mode type), robustness and difficulty of fabrication. We have described the design process itself as well as the optimization with respect to the rf transmission, taking into account mechanical limitations throughout. A detailed sensitivity investigation has been presented to emphasize the advantage of a double notch filter on the basis of which the PROBE V3 and HOOK V3 couplers are favored. They do not need any tuning system for the notch filter as they are robust enough against flange tolerances and even against thermal contraction during cool-down [35]. Subsequently, both couplers were slightly modified in order to suppress multipacting in the relevant field range while keeping the rf characteristics.

The thermal behavior of all couplers is very similar and dominated by the heat transfer through the rf cable rather than by the electromagnetic field inside the cavity. A thermalization at $2 \mathrm{~K}$ on the coupler tube and further heat intercepts on the rf cable are required to sufficiently reduce the thermal load.

Finally, a 3D-printed prototype of the PROBE V3 design was tested on a copper SPL cavity. The low power measurements at room temperature essentially confirmed the simulated results.

\section{ACKNOWLEDGMENTS}

The author would like to acknowledge the fruitful discussions with Thomas Flisgen. This work is sponsored by the Wolfgang Gentner Programme of the Federal Ministry of Education and Research.

[1] F. Gerigk, S. Atieh, I. Aviles Santillana, W. Bartmann et al., Conceptual Design of the Low-Power and High-Power SPL: A Superconducting $H^{-}$Linac at CERN (CERN, Geneva, 2014).
[2] S. Kim, SNS superconducting linac operational experience and upgrade path, in Proceedings of LINACO8, Victoria (JACoW, Geneva, 2008), p. 11.

[3] B. Aune, R. Bandelmann, D. Bloess, B. Bonin et al., Superconducting TESLA cavities, Phys. Rev. ST Accel. Beams 3, 092001 (2000).

[4] M. Schuh, F. Gerigk, J. Tückmantel, and C. P. Welsch, Influence of higher order modes on the beam stability in the high power superconducting proton linac, Phys. Rev. ST Accel. Beams 14, 051001 (2011).

[5] M. Schuh, Ph.D. thesis, Inst. Appl. Math., Heidelberg, 2011.

[6] C. Liu, W. F. O. Müller, W. Ackermann, and T. Weiland, CERN Technical Report No. sLHC-Project-Note-0040, 2012.

[7] H. Padamsee, T. Hays, and J. Knobloch, RF Superconductivity for Accelerators (Wiley, New York, 1998), p. 66, 134.

[8] M. Schuh and W. Weingarten, CERN Technical Report No. sLHC-Project-Note-0027, 2010.

[9] D. Kajfez, Encyclopedia of $R F$ and Microwave Engineering (John Wiley \& Sons, New York, 2005), p. 3937.

[10] J. Eberhardt, CERN Technical Report No. CERN-ACCNOTE-2014-0009, 2014.

[11] T. Flisgen, H.-W. Glock, P. Zhang, I. R. R. Shinton, N. Baboi, R. M. Jones, and Ursula van Rienen, Scattering parameters of the $3.9 \mathrm{GHz}$ accelerating module in a freeelectron laser linac: A rigorous comparison between simulations and measurements, Phys. Rev. ST Accel. Beams 17, 022003 (2014).

[12] CST-Computer Simulation Technology Ver. 2016 (CST AG, Darmstadt, Germany, 2016).

[13] K. Papke, F. Gerigk, and U. van Rienen, HOM couplers for CERN SPL cavities, in Proceeding of SRF2015, Paris (JACoW, Geneva, 2015) pre-pressed MOPB078.

[14] B. P. Xiao, S. A. Belomestnykh, I. Ben-Zvi, J. Skaritka et al., Compact higher order mode filter for crab cavities in the large hadron collider, in Proceeding of SRF2013, Paris (JACoW, Geneva, 2013), p. 1006.

[15] E. Haebel, V. Rödel, F. Gerigk, and Z. T. Zhao, The higherorder mode dampers of the $400 \mathrm{MHz}$ superconducting LHC cavities, in Proceeding of 8th Workshop on RF Superconductivity, Abano Terme (JACoW, Geneva, 1997), p. 701.

[16] F. Gerigk, The higher-order mode dampers of the $400 \mathrm{MHz}$ superconducting LHC cavities (unpublished).

[17] HFSS—High Frequency Structural Simulator Ver. 2014 (ANSYS Inc., Pennsylvania, 2014).

[18] S. Molloy, R. Ainsworth, and R. Ruber, Multipacting analysis for the superconducting RF cavity HOM couplers in ESS, in Proceedings of the 2nd International Particle Accelerator Conference, San Sebastiáán, Spain (EPS-AG, Spain, 2011), p. 190.

[19] A. Sun, Y.-Z. Tang, L.-P. Zhang, and Y.-M. Li, A coaxial HOM coupler for a superconducting RF cavity and its low-power measurement results, Chin. Phys. C 35, 301 (2011).

[20] G. Wu, E. Harms, and T. Khabiboulline, FermiLab Technical Report No. FNAL-TD-08-019. 
[21] D. C. Mattis and J. Bardeen, Theory of the anomalous skin effect in normal and superconducting metals, Phys. Rev. 111, 412 (1958).

[22] T. Junginger, Ph.D. thesis, Heidelberg U., 2012.

[23] J. Halbritter, Institut fuer Experimentelle Kernphysik Technical Report No. 3/70-6, 1970.

[24] G. Ciovati, R. Geng, J. Mammosser, and J. Saunders, Residual Resistance Data From Cavity Production Projects at Jefferson Lab, IEEE Trans. Appl. Supercond. 21, 1914 (2011).

[25] CryoComp Rapid Cryogenic Design (Eckels Engineering Inc., 2011), http://www.eckelsengineering.com/.

[26] G. Ciovati, Thomas Jefferson Lab National Accelerator Facility Technical Report No. JLAB-TN-03-003, 2003.

[27] C. E. Reece, E. F. Daly, G. K. Davis, W. R. Hicks et al., Diagnosis, analysis, and resolution of thermal stability issues with HOM couplers on prototype CEBAF SRF cavities, in Proceedings of SRF2007, Beijing (JACoW, Geneva, 2007), p. 540.

[28] W. Henneberg, R. Orthuber, and E. Steudel, Zur Wirkungsweise des Elektronenvervielfachers, Z. Tech. Phys. 17, 115 (1936).
[29] K. Ko and A. Candel, in Proceedings of the 25th International Linear Accelerator Conference, LINAC2010, Tsukuba, Japan (KEK, Tsukuba, Japan, 2010), p. 1028.

[30] C.-K. Ng, L. Ge, C. Ko, Z. Li, and L. Xiao, Multipacting simulation of accelerator cavities using ACE3P, in Proceedings of the 25th Particle Accelerator Conference, PAC2013, Pasadena, CA, 2013 (IEEE, New York, 2013), p. 216.

[31] R. Ainsworth, R. Calaga, and S. Molloy, HOM coupler optimisation for the superconducting RF cavities in ESS, in Proceedings of the 3rd International Particle Accelerator Conference, New Orleans, LA, 2012 (IEEE, Piscataway, NJ, 2012), p. 2182.

[32] G. Romanov, FermiLab Technical Report No. FERMILABPUB-11-003-TD, 2011.

[33] K. Papke, F. Gerigk, and U. van Rienen, HOM couplers for CERN SPL cavities, in Proceeding of SRF2013, Paris (JACoW, Geneva, 2013), p. 1066.

[34] B. Gustavsen and A. Semlyen, Rational approximation of frequency domain responses by vector fitting, IEEE Transactions on Power Delivery 14, 1052 (1999).

[35] K. Papke, CERN Technical Report No. sLHC-ProjectNote-0044, 2016. 